\title{
Article \\ Electrophilic Aromatic Substitution: theoretical insight into halonium ion self-existence in catalytic system
}

\author{
Nikola Stamenković ${ }^{1}$ and Janez Cerkovnik ${ }^{2, *}$ \\ 1 University of Ljubljana, Biotechnical Faculty, Department of Food Science and Technology, Jamnikarjeva \\ 101, 1000 Ljubljana, Slovenia \\ 2 University of Ljubljana, Faculty of Chemistry and Chemical Technology, Department of Chemistry and \\ Biochemistry, Več na pot 113, 1000 Ljubljana, Slovenia. \\ * Correspondence: janez.cerkovnik@fkkt.uni-lj.si
}

\begin{abstract}
Potential Energy Scan (PES) has already proven to be a powerful tool in computational chemistry to detect critical points in the energy path of a system, such as transition states and local minima/maxima in energy convergence. Previous studies showed a wide application of PES in many different fields of physical-chemical sciences, such as materials, supramolecular, and catalysis chemistry. Moreover, the evaluation of the basic PES algorithms at a reasonably affordable level of theory has in principle revealed good basic statistical relationships that allow further investigations in this research area. Herein, a simple and fast graphical method for accurate PES evaluation was proposed, performed at the PM7 semiempirical level of theory for catalytic systems in electrophilic aromatic substitution processes. The results presented in this case study showed a relative error ranging from 1.5 to $27.1 \%$ for most catalytic-electrophiloid systems. The treatment of such systems with PES algorithms led to novel iron(V) species and opened a completely new field in tandem transition metal-nonmetal catalysis, implying entirely new insights. Moreover, the basic statistical analysis showed that there are no significant outliers, and therefore it can be concluded that the graphical analysis approach can be used in further detailed treatment of PES results in the search for saddle points and prediction of transition state properties under known conditions in the DFT and MP2 functions discussed here. The novel graphical methodology has been introduced by two applied graphical methods, and its accuracy demonstrated in semiempirical methods provides solid results in view of future development and application in a wide range of chemical sciences.
\end{abstract}

Keywords: Second-Order Derivative (SOD) method; Graphic method; Potential Energy Scan (PES); Charge-Transfer complex; Iron(III) bromide; Iron(V) complex; Transition metal-halogen tandem catalysis; Halonium ion

\section{Introduction}

Electrophilic aromatic substitution (EAS) is the most discussed process in the chemical community, along with oxidations, reductions, and bond activation processes. So far, various mechanistic analyzes have been carried out and different mechanism pathways have been identified [1-3], especially with the development of new instrumental techniques, such as infrared (IR) spectroscopies with time-dependent resolution structure (i.e. femto- IR spectroscopy) or laser-induced IR [4]. On the other hand, catalysis, as a widely known and indispensable branch of chemical science nowadays, is undergoing a great development in the organometallic framework, especially considering transition metal chemistry therein [5]. Interestingly, many catalytically optimized systems for EAS have been reported so far, ranging from pro-electrophilic to protonic systems $[6,7]$. The 
choice of electrophile in EAS has also been established as an important guideline in the optimization of potential new catalytic systems, and this is also a kind of answer to the unspeakable question why there are so many different catalytic "solutions" nowadays.

1.1. Theoretical aspects of the physicochemical outcome of interactivity patterns in general and EAS chemistry

The chemistry behind EAS depends strictly on the choice of the range of catalytic and electrophilic systems. Electrophiles discussed within EAS theory are somewhat more chemically subtle than typical electrophiles used in materials syntheses or even total syntheses, and can be ascribed as multiple species that are usually in tandem arrangement. Moreover, novel electrophiles are optimized to resemble the tandem reactivity pattern not only among themselves (i.e., they are not dimers or polymers, in the so-called intra-reactivity setup), but also within the catalytic-electrophilic reactivity type (so-called inter-reactivity setup). From a mechanistic point of view, the first reactivity pattern can be referred to as an autocatalytic reactivity pattern and the second as a heterogeneous catalytic reactivity pattern. The choice of the nucleophile is limited to the presence/absence of a functional group on the aromatic compound.

The nature of aromatic compounds has already been discussed in terms of the $\pi$ electrons responsible for the initiation process in EAS. Note that, in particular, $\pi$-electrons are (much) more mobile than corresponding $\sigma$-electrons, which leads directly to the key idea that the kinetic energies of $\pi$-electrons (also called $\pi$-kinetic energies) are gradually higher than those of corresponding $\sigma$-electrons. Moreover, due to the higher values of the kinetic energy of $\pi$-electrons, the mobility gradient analysis can show that there is a small discrepancy within the different types of $\pi$-electrons based on their topological definition [8]. The analysis of orbital shifts [9], on the other hand, can be a very striking and efficient tool in the tracking of $\pi$-electrons, suggesting that it is more than a phenomenon of increased electron mobility. Indeed, (fast) electron displacement in a given space is called a resonance phenomenon. In contrast, $\sigma$-electrons are axially bound to an atom, which prevents significant electron shift. Their $\sigma$-kinetic energies are lower than the $\pi$-kinetic energies because the space in which they can be located is significantly reduced. The resulting sum of the $\pi$ - and $\sigma$-kinetic energies can be viewed as a simple mathematical relation called the total kinetic energy of a system (eq. 1), and their difference can be simply referred to as a kind of Electron to Space Interaction factor (ESI, eqs. 2a and $2 b$ ):

$$
\begin{aligned}
& E_{k}=E_{\pi}+E_{\sigma} \\
& E S I=E_{\pi}-E_{\sigma}
\end{aligned}
$$

Since in aromatic compounds E_ $\pi \gg E_{-} \sigma$ is a valid approximation, ESI can be approximated

$$
E S I_{A r} \sim E_{\pi}
$$

Where $\mathrm{E}_{\pi}$ is referred to here as the $\pi$-kinetic energy and $\mathrm{E}_{\sigma}$ as the $\sigma$-kinetic energy. ESI is a newly introduced quantity that can predict trends in macroscopic properties - binding energies and physical characterization of systems with highly functionalized topologies on the one hand, and microscopic properties - consistent differentiation of zero-point energies, entropy gradients, and definitions of the localized electron density function - on the other [10]. In given aromatic systems, ESI can be applied specifically with respect to Negative Electrostatic Potential Map (NEPM) and thus predict the volume and electron density, which provides the opportunity to determine the electron density gradient, which can be important for the study of both binding processes and principles of reactivity in solution. Figure 1 shows the location of the NEPM over typical substituted 
monoaromatic compounds, which may correspond to an (increasing) ability in binding forces to eventually interpret the binding scheme between such compounds.

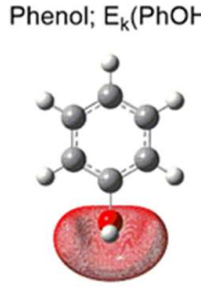

(a1)

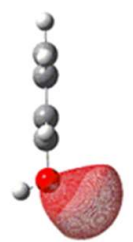

(a2)

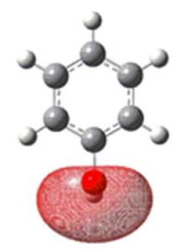

(a3)

Aniline; $E_{k}\left(P h N H_{2}\right)=286.4079$ a.u.

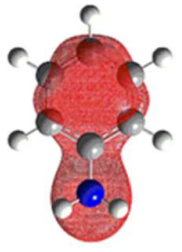

(b1)

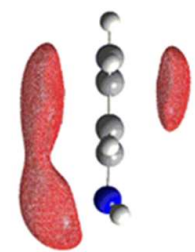

(b2)

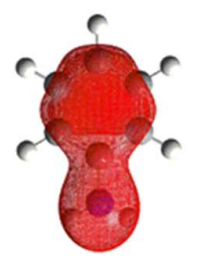

(b3)

Phenylborane; $E_{k}\left(\mathrm{PhBH}_{2}\right)=256.5476$ a.u.

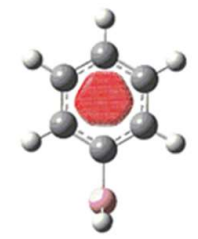

(c1)

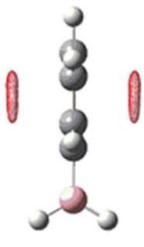

(c2)

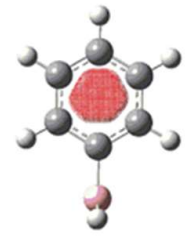

(c3)

Phenyllithium; $E_{k}(P h L i)=238.1067$ a.u.

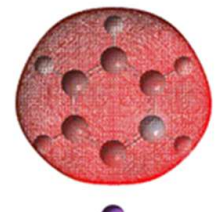

(d1)

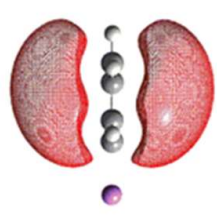

(d2)

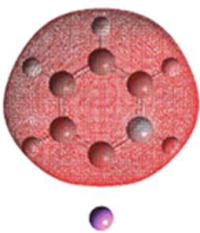

(d3)

Figure 1. NEPM calculated for some simple monosubstituted aromatic compounds. The partial maps shown in structures 1 and 3 mainly indicate where the negative charge is concentrated, and therefore suggest where on the aromatic compound the binding process is most likely to start. Structures 2 show an important feature of this type of map - the density level. NEPM can be used to predict positions in EAS with relevant qualitative accuracy, as well as to predict electronic effects of substituents. Further analogies can be derived for other types of compounds. The kinetic energies (Ek) of aromatic compounds depend on the type and nature of the substituent, i.e., from phenol ( $\pi$-electrons are most mobile) to phenyllithium ( $\pi$-electrons are concentrated over negatively charged ipso carbons, which are least mobile). On the other hand, phenyllithium is the most reactive in both kinetic and thermodynamic sense, while phenylborane is the least reactive due to the orbital arrangement on the molecular map. NEPM can determine the mobility of $\mathrm{n}$ - and $\pi$-electrons since their physicochemical nature is similar, unlike $\sigma$-electrons, which is reflected in the $\mathrm{n}$ - and $\pi$-mobility coefficients, which are (much) larger than $\sigma$-electrons. All calculations were performed at the M062X/Def2QZVPPD//M062X/6-311G(2df,2pd) level of theory.

Although the kinetic energy of an electron is an important element in the evaluation methodology of binding processes, there is another factor that additionally determines 
the total energy of an electron in a given state - the potential energy. The potential energy refers to the interactions between electrons and can be represented as either a Coulomb or an overlap integral. These integrals ultimately yield a value, theoretically represented as a density matrix, from which one can read whether and how strongly the electrons interact from an electrostatic point of view. The larger the value of a matrix - the better the coverage. The sum of the contributions of potential and kinetic energy is equal to the total energy of a system (electronic energy of a system), represented in eq. 3:

$$
E_{\text {total }}=E_{k}+E_{p}
$$

Here $E_{t o t a l}$ is called the total (electronic) energy of a system, $E_{k}$ is the kinetic energy and $E_{p}$ is the potential energy. If we take into account the presence of $\sigma$-and $\pi$-electrons, which contribute (significantly) to the modified reactivity in EAS or other types of retrons [10,11], eq. 3 can be extended to eq. 4 :

$$
E_{\text {total }}=E_{\pi}+E_{\sigma}+E_{p}
$$

So there remains the space to define the mathematical meaning of the kinetic energy of an electron, as a particle. As free $\pi$-electrons can move between nearby orbitals, shift their position and maintain momentum over time, which confirms the Heisenberg relation as constant, since the velocity of an electron as a particle reaches values much smaller than the speed of light, which is shown in the following equations as follows [12]:

Combining Heisenberg basic postulate (eq. 5) with the relativistic momentum equation in the formalism of classical mechanics (eq. 6), one obtains the velocity-modified Heisenberg principle (eq. 7), which refers to the kinetic property of a particle (e.g. electron):

$$
\begin{aligned}
& \Delta \sigma_{x} \Delta \sigma_{p} \geq \frac{\hbar}{2} \\
& \Delta p_{e}=m_{e} \Delta v_{e} \\
& \Delta \sigma_{x_{e}} \Delta \sigma_{v_{e}} \geq \frac{\hbar}{2 m_{e}}
\end{aligned}
$$

Where $\Delta \sigma_{x_{e}}$ is the momentum value of an electron at a given time reference function, $\Delta \sigma_{v_{e}}$ is the velocity momentum value at a given time reference function, where me and $\Delta v_{e}$ denote the electron mass (in $\mathrm{kg}$ ) and velocity change (in $\mathrm{ms}^{-1}$ ), respectively. Reduce the eventual probability of electron motion within a narrow electron distance (cf. reduced Planck constant to a full single frequency corresponding to $2 \pi$, or radius distance that can be approximated for a spin [electron]). Furthermore, if the velocity of the $\pi$-electrons is assumed to be constant, eq. 7 can be simplified in terms of the kinetic velocity of the electrons, further defining eq. 7 :

$$
\begin{aligned}
& \Delta E_{k}(e)=\frac{1}{2} m_{e} \Delta v_{e}^{2} \\
& \Delta \sigma_{v_{e}}=\alpha \Delta v_{e}=\alpha \sqrt{\frac{2 \Delta E_{k}(e)}{m_{e}}} \\
& \Delta \sigma_{x_{e}} \alpha \sqrt{\frac{2 \Delta E_{k}(e)}{m_{e}}} \geq \frac{\hbar}{2 m_{e}} \\
& \Delta \sigma_{x_{e}} \geq \frac{\hbar}{\alpha} \sqrt{\frac{1}{8 \Delta E_{k}(e)}}
\end{aligned}
$$

Here $\Delta E_{k}(e)$ is denoted as the change in the kinetic energy of the electron solely as a function of the kinetic velocity of the electron, and $\alpha$ is a term corresponding to the 
frequency of change in electron velocity, which is inversely proportional to the frequency of collision with other electrons (in systems with many electrons) and the medium in which the electron is located. If the electron is in the axial bond orbital, then the value of the density overlap matrix is assumed to be high, which means that the value of the Coulomb integral is also increased. Since the electrons in the bonding orbital repel each other, the orbitals themselves must have opposite sign to attract each other for the electrons to move in the given overlap space. On the other hand, if an electron is in a secondary bonding orbital, the density matrix can vary depending on the type of orbital and the number of electrons in it. Usually, the value of the density overlap matrix in this case is a time-dependent quantity because the value of the Coulomb integral is smaller than for axial bond orbitals and the electrons have the opportunity to diffuse through the multiply occupied space. Since the orbital is defined as the square of the probability that an electron is at a particular point in the system "x", defined via the wave equation formulated in the Schrödinger equation [10,13], it can be postulated that the momentum of an electron can be determined as the limiting value(s) of the space traversed by the electron, as a function of time and position, defined as in Equation 12:

$$
\begin{aligned}
& \Delta \sigma_{x_{e}}=\lim _{\substack{n \rightarrow L \\
k \rightarrow r_{\text {max }}^{L}}} \sum_{i=0}^{n} \sum_{j=0}^{k} c_{i} \psi^{2} c_{j} r_{\psi^{2}}|x, y, z, t\rangle \\
& r_{\psi^{2}}=\sqrt{\sum_{x, y, z}(\Delta x)^{2}(\Delta y)^{2}(\Delta z)^{2}} \oint_{V} \psi^{2} d \psi
\end{aligned}
$$

Here $r_{\psi^{2}}$ is defined as the eigenvector of an orbital containing an electron, and is defined as a contour integral as a function of the volume of the corresponding orbital described by the corresponding wave function $\psi$, and its coordinate number set described as a sum of linear vector functions by the $\mathrm{x}, \mathrm{y}$, and $\mathrm{z}$ coordinates. $r_{\text {max }}^{L}$ is defined as the most intense eigenvector at the given value of $\mathrm{L}$. $\mathrm{L}$ is the angular momentum of the analysed electron energy, while $c_{i}$ and $c_{j}$ are electron and orbital coefficients, respectively. Assigning physical meanings to eqs. 12 and 13 , one can conclude that $c_{j}$ is proportional to the orbital energy level, i.e., it defines the frontier molecular orbital image of an analysed system containing a given electron(s). Moreover, by solving eq. 12, it is a simple observation that the comparison of the solutions for $\pi$ - and $\sigma$-electrons of the same quantum level indicates that $\Delta \sigma_{x_{e}}$ is larger for $\pi$-electrons than for $\sigma$-electrons, which also suggests that $\Delta \sigma_{x_{e}}$ can be considered as a parameter of electron mobility.

\subsection{Energy as a function of Electron Mobility parameter in PES theory}

Energy is a ubiquitous phenomenon when it comes to the physical aspect of a system and can be represented as an internal driving force. When considering binding processes, only the energy of the relevant electrons is considered, and the energy of the system in such a transition state (here the terms transition and transition states are disambiguated, the former denoting any process in which the electron density is minimally perturbed as a function of orbital and geometrical properties and time; and the latter - denotes the exact electron movement from one to another reactive site, which brings the interacting subsystems out of equilibrium, usually leading to quite an increase in energy, which can be diagnosed by analysing the density overlap matrices, which have only an imaginary frequency), increased by the entropy decrease. This type of energy (electronic energy) is thus responsible for the stability of the whole system and its chemical reactivity, and is essentially composed of a thermodynamic parameter (enthalpy) and a physical parameter (work). When chemical interactions are taken into account, another additional but important factor can be the geometrical parameter (entropy) of the subsystem, which is additionally determined by kinetic analyses.

PES analyses are a widely used theoretical tool in the study of transition states (TS) [14] and is a fundamental method for other modern algorithms at TS search paths, such 
as Nudged Elastic Band (NEB) theory [15], Quadratic Synchronous Transit methods (QST) [16], Linear Synchronous Transit (LST), Berny's approximation algorithm, and Synchronous Transit-Guided Quasi Newton method (STQN). PES analyses are invoked by defining relaxed scan reaction coordinates. This scan can be defined on one or more reaction coordinates at a time, which defines the ability for the prediction algorithm to create the potential energy surface on one coordinate (1D), two coordinates (2D), or three coordinates (3D). 3D potential energy surfaces are the most time-consuming to create, but are the most accurate and are better suited for predicting systems with multiple reactive sites than for systems with only one reactive site (e.g., reactive methyl compounds). Nevertheless, the ratio of predicted relative errors to time required is much better for 1D scans than for 3D scans, and - assuming a good theory used in these algorithms - 1D scans can be quite good even for large and complex reactive systems with up to several hundred atoms involved. In a theoretical sense, when using methods based on the wave function or electron density integral, the energy change of a system along the chosen reaction coordinate should follow a uniform energy change, which in turn depends strongly on the proximity of electrons defined by both the bond eigenvectors and the map of the uniform molecular electrostatic potential.

Assuming that the electronic energy is equal to Hamilton's equation, eq. 12 can be rewritten as follows as a starting point, since it is a time-independent Schrödinger equation:

$$
\widehat{H}=\widehat{T}+\widehat{V}
$$

If $\hat{T}$ is a kinetic energy operator and $\hat{V}$ is a potential energy operator, then the Hamiltonian operator is equal to the electronic energy as given earlier in eq. 3 . The kinetic energy operator can be further transformed into a relation defined by the momentum coordinate:

$$
\begin{aligned}
& \widehat{T}=-\frac{\hbar^{2}}{2 m} \nabla^{2}=-\frac{\hbar^{2}}{2 m}\left(\frac{\partial^{2} \psi}{\partial x^{2}}+\frac{\partial^{2} \psi}{\partial y^{2}}+\frac{\partial^{2} \psi}{\partial z^{2}}\right) \\
& \widehat{V}=E_{p}=\hat{V}(r, t)
\end{aligned}
$$

Where $r$ and $t$ are the corresponding eigenvector and time period defining the intensity of the electron potential energy, further defined by the wave equation or electron density integral. When the number of reaction coordinates is reduced from three to one, the computational cost is also reduced proportionally, since the mathematical complexity of calculating the derivative is reduced, as can be seen from eq. 15, and thus the Hessian can be calculated more quickly:

$\widehat{T}=-\frac{\hbar^{2}}{2 m} \nabla^{2}=-\frac{\hbar^{2}}{2 m} \frac{\partial^{2} \psi}{\partial x^{2}} \quad$ (kinetic operator form in one-dimensional PES method)

Eq. 13 remains intact since the orbital eigenvector is strictly defined in the Cartesian coordinate system, where all three coordinate vector intensities are known, due to the orbital displacement in space. On the other hand, Electron Mobility parameter can be considered according to the two basic definitions of Schrödinger equation, which allows us to exclude the time-independent form of eq. 18a:

$$
\Delta \sigma_{x_{e}}=\lim _{\substack{n \rightarrow L \\ k \rightarrow r_{\text {max }}^{L}}} \sum_{i=0}^{n} \sum_{j=0}^{k} c_{i} \psi^{2} c_{j} r_{\psi^{2}} \frac{\partial}{\partial x}|y, z\rangle
$$

The degree of freedom is also involved in the computational procedure and may also dictate the total computational time with respect to the possible structures analysed along the chosen reaction coordinate. Furthermore, it defines to what extent the reaction 
coordinates are involved in the further energy definition in the method PES and how the symmetry element affects the behaviour of the algorithm of the method PES as a function of time.

\subsection{Energy path change along reaction coordinate - Cartesian Displacement Restriction phenom-} enon

As already shown in eq. 14 with reference to eq. 3, electronic energy correlates proportionally when transposed into a vector system, which opens the possibility of expressing the change in energy gradient as a function of coordinate change. Each coordinate change is directly associated with small energy values added to the potential energy, which is an intrinsic property of an electron, and assigned to the simple vector of kinetic energy. Even though Schrödinger's atomic theory surpasses Bohr's, certain postulates in the interpretation of quantum mechanics remain valid to this day. One of these is that the electron, with its kinetic energy determined by its internal distance from the nucleus and its position at the predicted orbital level (position and state in a subshell), is defined such that its probability of being found in a space is determined by the normalized wave function $\psi^{2}$, this probability being increased up to $99 \%$ if both phases of the wave function are squared, thus covering the entire range of the function. Since the electron exerts a certain amount of kinetic energy, it is given a certain trajectory in space. This trajectory is complex enough to rationalize not only as a simple vector value in an imposed imaginary coordinate system consisting of $\mathrm{x}, \mathrm{y}$, and $\mathrm{z}$ coordinates, but as $\mathrm{n}$ vector values defined as a function of time and energy. On the other hand, in terms of the whole system in which analyzed electron motion is assumed to be a stationary point in this type of analysis, any minimal motion of such an electron will cause increase in kinetic energy and decrease in potential energy, which will eventually result in energy and motion gradients increase. These two gradients are defined as partial increase as a function of time, thus defining the energy operator $\hat{E}$, as follows:

$$
\hat{E}=\left(\frac{\partial}{\partial x}+\frac{\partial}{\partial y}+\frac{\partial}{\partial z}\right)_{t} E_{k}
$$

For the physical meaning, a further derivation is required to specify the exact dependence of the energy gradient on any coordinate at a given time, leading to a form similar to the Laplacian operator $\nabla^{2}$ :

$$
\hat{E}^{2}=\left(\frac{\partial^{2}}{\partial x^{2}}+\frac{\partial^{2}}{\partial y^{2}}+\frac{\partial^{2}}{\partial z^{2}}\right)_{t} E_{k}=\nabla^{2} E_{k}
$$

For a single coordinate, such as the chosen reaction coordinate, eq. 20 reduces to a oneparameter Laplacian operator, resulting in a second-order kinetic energy gradient:

$$
\hat{E}_{x}^{2}=\left(\frac{\partial^{2} E_{k}}{\partial x^{2}}\right)_{t, y, z}
$$

When applied to predicting the energy path of such an electron in a given system, the only physical solution to eqs. 20 and 21 is the change in energy gradient, which is almost always positive, due to the increase in kinetic energy. The resulting fact causes the system to cancel the incoming energy increase in a slight system shift. In addition to the energy change, this shift also causes a change in position of each reactive site and the entire system, which can be said to be prohibitive due to the integrity of the eigenvector in the ground state. This prohibition of the position change leads to a change in the symmetry of the whole analysed system, which means a slight positive energy change. This change can alter the result of PES itself, where such a change can be considered as a kind of outlier. This outlier is a limiting condition for the energy gradient to remain 
constant in the PES algorithm, which can be referred to as the phenomenon or effect of Cartesian Displacement Restriction (CDR).

The CDR effect can be defined mathematically as the sum of the energy gradient perturbation as a function of the position parameters, i.e. the change in Cartesian coordinates expressed in their vector form:

$$
\sum_{r_{x}, r_{y}, r_{z}}\left(\frac{\partial E_{C D R}}{\partial t}\right)=E_{0}+\sum_{n=1}^{m} E_{n}=E_{0}+\sum_{n=1}^{m} \Delta U+p \Delta V
$$

Where $n$ and $m$ are denoted as the initial and final number of microstates that a system passes through during the estimation of the PES algorithm, depending on a chosen reaction coordinate. Alternatively, since electronic energy can refer to the Heat of Formation $(\mathrm{HoF})$ of a system, it can be extended to the internal energy $(\Delta U)$ of the system and the work $(p \Delta V)$ it exerts on all given number of electrons as a function of aiven number of microstates. The result of the work exerted on the system always leads to a positive energy change, so CDR can usually be considered as a positive value. CDR may occur one or more times during the computation of the algorithm PES, and the number of CDRs may be functionally correlated with the level of theory chosen for such PES estimation. Moreover, the mathematical analysis in terms of the derivation theory may be suitably correlated with various types of transition state predictions from simple results obtained by the PES algorithm implementation which are to be presented.

\section{Results and discussion}

The results to be presented here were obtained at the PM7 semiempirical theory level, which was chosen as the optimal choice of theory level due to the optimization parameters and the set of atoms for which it was optimized [18]. Scans were performed for monomeric iron(III)bromide ( $\mathrm{FeBr}$ ) on electrophilic halogen species for $\mathrm{F}, \mathrm{Cl}$, Br and I. The reason for choosing these two compounds as catalytic species is their applicability under experimental conditions.

"Electrophiloid" here is a new term for species that provide electrophile during the EAS process. Electrophiloids are usually of covalent character or ion pairs, so their use does not necessarily explain the presence of electrophile in this type of process. However, in the presence of a catalytic species, the electrophilic compound should be able to interact and form a charge-transfer complex (CT) that allows the electrophiloid to be attacked by a nucleophilic aromatic nucleus [2,3]. This attack leads to geometry changes and an increase in energy. The energy increase occurs through the formation of a $\pi$-complex, where $\pi$-electrons are bound to the CT -complex, which increases the electron mobility parameter and thus the frequency of collisions ( $\alpha$ in eqs. 10 and 11). Moreover, interesting binding states can be observed in an energy profile, albeit with (very) low energy, as a function of time and reaction coordinate, which clearly means that corresponding transition states can be detected. To our knowledge, this is the first time that the graphical method has been used to identify this type of transition state. The electrophiloids used in this work were selected rather according to their commercial availability and/or experimental suitability, with the exception of the fluoro electrophiloid. Therefore, the compounds were selected as follows: oxygen(II) difluoride $\left(\mathrm{F}_{2} \mathrm{O}\right)$ as fluorinating agent, chlorine $\left(\mathrm{Cl}_{2}\right)$ as chlorinating agent, bromine $\left(\mathrm{Br}_{2}\right)$ as brominating agent and iodine $(\mathrm{I})$ chloride $(\mathrm{ICl})$ as iodinating agent. Other, more favorable fluorinating agents were found [19], but none of them were expected to interact in a Lewis acid-like catalytic manner to fluorinate aromatic compounds, so they were excluded from this study.

\subsection{Introduction to Graphic method analysis}

Mathematical algorithms have already proven useful in designing potential energy scans that allow simple or multivariable monitoring of energy change as a function of time. Now we can use the same mathematical apparatus to monitor energy changes much 
smaller than those for "standard" transition states, as mentioned earlier. Analysing even the simplest (1D) potential energy scan of various systems, from inorganic to organic, one can come up with the idea that any plot of energy vs. reaction coordinate contains points that can be characterised as an unbounded function. The unbounded function can be further analysed by differentiating or integrating it to characterise one or more peaks that are subject to further scientific interpretation. The situation becomes simpler if we consider that almost every chemical system follows the same mathematical pattern that predicts the increase of the energy of the whole system when the density gradient is (strongly) changed by external conditions (i.e., violation of the electron density conservation law due to increased polarity of the interacting species, due to some physical or chemical changes induced by the environment) [20]. Moreover, any potential energy scan (whether 1D, 2D or 3D) can be predicted by applying a fitting algorithm to such plots, which provides a mathematical fitting equation for that plot line [21-23]. The accuracy of such fitting equations can be easily evaluated by analysing the Pearson correlation value or R2 value. In this and subsequent manuscripts, we will present the form of such 1D plots as well as their approximate fitting equation and criteria for their appropriate evaluation.

Differentiation as a computational procedure can be adequately used in PES plot analyzes and provides the precise point on such a plot at which the increase in the energy gradient is detected. As a given parallel to point determination, differentiation can also be used to predict the curvature point of a designed PES plot, i.e., to determine its inflection point (point on the graph/plot where the function changes sign). The results of first and second order derivatives of PES plots shown here have proven useful for graphical analysis, emphasizing that higher order derivatives can produce mirror image results to those of first or second order derivatives, leading us to generally discourage the use of higher order derivatives in this type of analysis.

\subsubsection{First order derivative (FOD) analysis}

This type of derivation is attributed to the change in the flow function, where a significant increase in the given values leads to the formation of a peak in the given function, described as follows:

$$
y^{\prime}(x)=\frac{y_{2}-y_{1}}{x_{2}-x_{1}}=\frac{\Delta y}{\Delta x}
$$

Or, if $\Delta y$ is a small value, we may assume that the first derivative of a function with respect to $y$ is considered as a differential value, i.e.:

$$
y^{\prime}(x)=\frac{d y}{d x}
$$

All potential energy scans are characterized by one or more peaks that determine energy deviations, as discussed briefly earlier (see above). Moreover, in general, these functions almost always resemble a sine, exponential, or polynomial function. This leads to the simple conclusion that the functions may have some global or local maxima/a or minima/a. These minima are characterized mathematically as points at which the flux function changes sign, and in functional analysis any change in the sign of a function can be attributed to a significant change, whether considered as a mathematical or chemical path. Normally, it is not possible to infer the exact nature of processes from the first derivative of a given function, as the methodology for identifying peaks is mathematically inadequate and therefore requires more precise mathematical definitions! Such definitions are solved with the introduction of the second derivative of a function, $y^{\prime \prime}$ $[24,25]$.

2.1.2. Second order derivative (SOD) analysis 
Second-order derivatives are much more commonly used in mathematical analysis because they are superior to first-order derivatives in scope and accuracy. In particular, for nonlinear functions, such as exponential or sinusoidal functions, second-order derivatives are well suited not only to explain the change in the flow function (i.e., the inflexion point), but also to perform more accurate analyses of chemical microstates. In fact, an additional first derivative of the first derivative can explain the determination of the curvature in a simpler way:

$$
y^{\prime \prime}=\frac{d y}{d x} \frac{d}{d x}=\frac{d^{2} y}{d x^{2}}
$$

Eq. 25 is the simplest representation of the second derivative of the function $y$ in which the variable $x$ is included. In the next step, differentiation of nonlinear functions, one can see that the plots still show a nonlinear curvature with one peak (if the function is unlimited ) or with several identified peaks (if the function is limited by one or more points that lie exclusively outside the range of the flow function [also called outliers]).

If we now analyse PES plots, an assumption from the previous discussion may emerge - the continuity of the flow function, as well as the physico-chemical significance of outliers. For 1D scans, one should only differentiate along the single reaction coordinate and plot the second derivative against the reaction coordinate. In general, one can also make 3D plots as a function of the second order of the given thermodynamic quantity and the reaction coordinate itself, but this would require more computational power and time. The main relationship between the number of coordinates and the second derivative can be described as follows according to eq. 26 :

$$
y^{\prime \prime}=\frac{d^{2} E}{d x^{2}}|x, y, z\rangle
$$

Eq. 26 can be extended to all three coordinates given either in scalar or vector form, so that by rearrangement we obtain eq. 27 :

$$
y^{\prime \prime}=\frac{d^{2} E}{d x^{2}}+\frac{d^{2} E}{d y^{2}}+\frac{d^{2} E}{d z^{2}}=E\left(\frac{d^{2}}{d x^{2}}+\frac{d^{2}}{d y^{2}}+\frac{d^{2}}{d z^{2}}\right)=\nabla^{2} E
$$

Where $\mathrm{E}$ is any thermodynamic quantity suitable for energy monitoring (e.g. enthalpy or Gibbs free energy as more appropriate, or probably chemical potential or entropy change). As can be seen from eq. 27, the energy change along the second derivative is quite closely related to the constraint on the coordinate motion described by the phenomenon CDR (see above), which directly indicates the presence of a single factor - the Laplacian operator. The Laplacian operator and the second derivative of the potential energy scan can be plotted directly in proportion, allowing the nature of the bond to be predicted along the reaction coordinates $(\Delta x)$ and time period $(\Delta t)$ simultaneously, which in turn would reduce in the future the complexity of the mathematical apparatus needed to predict the bond in terms of potential orbital and positioning problems. The advantage of generating the Laplacian density can be welcomed as an additional insight into the binding when none of the dispositive properties are available [26-29]. Since the Laplacian operator depends on the chosen thermodynamic parameter, it must be emphasized here that the Laplacian density may vary with the available CDRs in a given analyzed system.

In this work, we will present the behavior of the second-order derivative method on the systems that are likely to be more difficult to study in the framework of electrophilic aromatic substitution theory due to weak non-covalent interactions, as well as provide insight into their bonding and find a broad transition state region. By simply constructing these diagrams, we can identify weak transition state boundaries by predicting less likely transition states, such as pre- and pseudo-transition states located around the true transition state. 


\subsection{FeBrs-Electrophiloid interaction in electrophilic aromatic substitution}

Iron(III) bromide ( $\left.\mathrm{FeBr}_{3}\right)$ is a water-soluble iron salt that is mostly covalently bonded to three bromine atoms with the $\mathrm{D}_{3 \mathrm{~h}}$ symmetry group and has a trigonal planar structure of the monomeric compound. The Fe-Br distance has been characterized at the theoretical level and shows a similarity of $2.269 \pm 0.010 \AA$ at the M06L/cc-pVTZ theory level, which agrees well with previously calculated Fe-Br lengths at the B3LYP theory level as well as at the PM3 semiempirical level [30]. Moreover, unlike the hypothetical Fe-Br bond order, which is slightly larger than 1, the predicted Wiberg bond indices are of lower order, ranging from 0.9 to 1 , with 0.9 predicted at M06L/cc-pVTZ. This slight decrease in bond order value may actually also reveal a slight ionic character within this compound, considering that the orbital picture may indicate additional information about the $\mathrm{Fe}-\mathrm{Br}$ bond strength. As a partially ionic compound, FeBr3 exists as a crystalline solid in both states, monomer or dimer [31,32]. Both types of compounds have been shown to be good Lewis acids, due to their high spin nature, which allows the construction of d orbitals (over $\mathrm{p}$ orbitals) as dominant among the LUMO orbitals, which extraordinarily emphasizes the importance of the $d_{z^{2}}$ LUMO orbital in showing the acidic nature of this compound. The sd hybridization of the LUMO orbital of $\mathrm{FeBr}_{3}$ at the Fe atom suggests the possibility of a wide range of nucleophilic accesses, mainly because of the specific eigenvector of the $d_{z^{2}}$ orbital with an orbital span of $180^{\circ}$. However, if one analyzes the eigenvector values of the LUMO orbital, one gets a better insight into the larger s-character of the FeBr3 LUMO, which competes well with $d_{z^{2}}$ as a LUMO, which in turn reduces the stereoelectronic advantage of nucleophilic accesses mentioned above. This makes the overall orbital picture less clear for the dominance of $d_{z^{2}}$ over the s orbital, which somewhat weakens the Lewis acidity compared to other Lewis acid-like compounds (e.g. $\mathrm{AlBr}_{3}, \mathrm{BF}_{3}$, etc.). However, $\mathrm{FeBr}_{3}$ can still act as a rather strong Lewis acid and even bind unpolarized electrophiloids to the Fe center $[33,34]$.

\subsubsection{Oxygen(II) fluoride bound to $\mathrm{FeBr}_{3}$}

Oxygen(II) fluoride $\left(\mathrm{OF}_{2}\right)$ is a colorless, malodorous gas at room temperature with a relatively increased density for a species in the gaseous state $(\approx 1.88 \mathrm{~g} / \mathrm{mL})$. $\mathrm{OF}_{2}$ can be evaluated as a rather strong oxidant when its chemical profile is analyzed, since two fluorine atoms are bonded to a single oxygen atom, which is in the +2 oxidation state. Naturally, $\mathrm{OF}_{2}$ is treated as a strong oxidant and tends to go from the +2 charge to the regular -2 charge of the oxygen atom, thus accepting a total of 4 electrons via a halfreaction described as follows:

$$
O F_{2(g)}+2 H_{(a q)}^{+}+4 e^{-} \rightleftharpoons 2 F^{-}+H_{2} O
$$

This represents its strong oxidizing power. Moreover, its standard electrode potential $\left(E^{\circ}=\right.$ $2.168 \mathrm{~V}$ ) [35] indicates a strong ability to interact oxidatively with other species, especially those whose $E^{\circ}$ is less than $2 \mathrm{~V}$. Moreover, its rather enhanced electron movement gradient $\left(\Delta \Delta \sigma_{e}=-1.208 \mathrm{mV} / \mathrm{K}\right)$ [35] also suggests an enhanced ability in oxidative processes, which can be remarkably demonstrated under experimental conditions by reacting also with strong bases along the same reaction pathway (e.g., with sodium hydroxide) [36]. The information available so far on $\mathrm{OF}_{2}$ raises the question of its reactivity model, whose reaction pathways are rather unpredictable.

An unusual ratio of oxygen to fluorine oxidation states also sheds light on another important reactivity pattern discovered in $\mathrm{OF}_{2}$. The decomposition pathway can possibly be attributed to first-order radical elimination (ER1), while the reactivity of this compound with other species can be better described by using the orbital analysis method. Indeed, it can be shown that the above-mentioned unusual ratio of oxidation states between oxygen and fluorine can be altered in favor of oxygen, especially with Lewis acids, which is not so uncanny considering that the terminal fluorine atoms are theoretically doubly electronically unstabilized via lateral, mostly non-covalent interactions. For example, a brief theoretical analysis performed for the AlBr3-F-OF system at the M06L/aug-cc-pVDZ 
level of theory (Figure 2) revealed an assumed bent fluoride interaction with oxygen and aluminum of $\angle \mathrm{O}_{5} \mathrm{~F}_{6} \mathrm{Al}=100.04^{\circ}$, which is only $3-5^{\circ}$ less than the ideal bent angle for sp ${ }^{3}$ oxygen atoms in $\mathrm{OF}_{2}$. Obviously, the bonded fluorine atom adopts the same hybridization pattern as oxygen, which was previously easy to assume. The only difference with oxygen is the fact that the bond orders of such a bonded fluorine are quite different from those of oxygen in $\mathrm{OF}_{2}$. Table 1 shows the Wiberg bond indices for $\mathrm{O}-\mathrm{F}$ bonds in $\mathrm{OF}_{2}$ alone and when bound to $\mathrm{AlBr}_{3}$ via a fluorine atom.

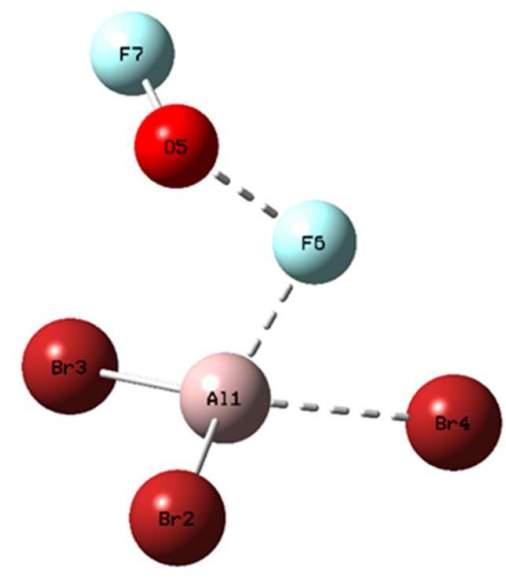

Figure 2. Optimized model of $\mathrm{OF}_{2}$ bound to $\mathrm{AlBr}_{3}$ via the fluoride atom in the charge-transfer (CT) complex. The aluminum adopts a bent tetrahedral geometry, with $\angle \mathrm{Br} 3 \mathrm{AlF} 6=111.56^{\circ}, \angle \mathrm{Br} 2 \mathrm{AlF} 6=111.42^{\circ}$, and $^{\circ} \angle \mathrm{Br} 4 \mathrm{AlF} 6=72.51^{\circ}$. Dashed bonds represent a bond order of less than 0.6, indicating that the bond lengths of $\mathrm{Al}-\mathrm{Br}(4)$ and $\mathrm{Al}-\mathrm{F}(6)$ are slightly increased due to the symmetry group change.

The thermodynamic analysis points out the disadvantage of binding the fluorine atom to the aluminium centre and describes the binding process as slightly endergonic, with a free energy barrier of up to $+10.642 \mathrm{kcal} / \mathrm{mol}$, which does not give a clear indication of whether or not this process agrees with the charge located on the fluorine atoms in $\mathrm{OF}_{2}$ (Table 2). Moreover, the NPA charges on $\mathrm{OF}_{2}$ and $\mathrm{AlBr}_{3}$ agree very well with the NPA charges on the AlBr3-F- OF complex. The partial positive charge on the fluorine atom (F6) in Figure 2 develops concertedly with the electron loss on the oxygen (which is again in agreement with the APT charge on the complex! [see Table 2]), which are partially stabilised via F-O backbonding interaction. Stability thermodynamic analyses were also performed at the same level of theory, confirming the slightly different result, but leading to what we believe to be a quite realistic picture of this binding process, which is described as a slightly exergonic process with $-2.436 \mathrm{kcal} / \mathrm{mol}$ total energy barrier.

Table 1. Bond lengths and Wiberg Bond Indices (WBI) at Mo6L/aug-cc-pvDZ level of theory for $\mathrm{AlBr}_{3}-\mathrm{F}-\mathrm{OF}$ complex

\begin{tabular}{ccccccc}
\hline & $\mathrm{F}(7)-\mathrm{O}(5)$ & $\mathrm{O}-\mathrm{F}(6)$ & $\mathrm{Al}-\mathrm{F}(6)$ & $\mathrm{Al}-\mathrm{Br}(2)$ & $\mathrm{Al}-\mathrm{Br}(3)$ & $\mathrm{Al}-\mathrm{Br}(4)$ \\
\hline Bond length $(\AA)$ & 1.353 & 1.869 & 1.811 & 2.258 & 2.259 & 2.396 \\
Wiberg Bond Index & 1.100 & 0.381 & 0.238 & 0.842 & 0.841 & 0.570 \\
\hline
\end{tabular}

Table 2. Natural Population Analysis (NPA), Mulliken and Atomic Polar Tensor (APT) charges on Mo6L/aug-cc-pvDZ level of theory for $\mathrm{AlBr}_{3}-\mathrm{F}-\mathrm{OF}$ complex.

\begin{tabular}{cccccccc}
\hline & $\mathrm{Al}$ & $\mathrm{O}$ & $\mathrm{F}(7)$ & $\mathrm{F}(6)$ & $\operatorname{Br}(2)$ & $\operatorname{Br}(3)$ & $\operatorname{Br}(4)$ \\
\hline $\begin{array}{c}\text { NPA charge } \\
\text { Mulliken }\end{array}$ & 1.362 & 0.083 & -0.031 & -0.430 & -0.446 & -0.443 & -0.096 \\
$\begin{array}{c}\text { charge } \\
\text { che }\end{array}$ & 1.121 & 0.223 & -0.204 & -0.263 & -0.360 & -0.345 & -0.171
\end{tabular}




\begin{tabular}{lllllllll} 
APT charge & 1.373 & 0.156 & -0.380 & 0.153 & -0.495 & -0.471 & -0.336 \\
\hline
\end{tabular}

Second, an analysis of the oxygen binding process to $\mathrm{AlBr}_{3}$ was performed to obtain a complete picture of the process exemplified here. For this purpose, under the same conditions ( $\mathrm{p}=1 \mathrm{~atm}, \mathrm{~T}=298.15 \mathrm{~K}$, considering toluene solvation interactions), we modeled and optimized the $\mathrm{AlBr}_{3}-\mathrm{OF}-\mathrm{F}$ system with outgoing Universal Force Field (UFF) molecular mechanics preoptimized structures.

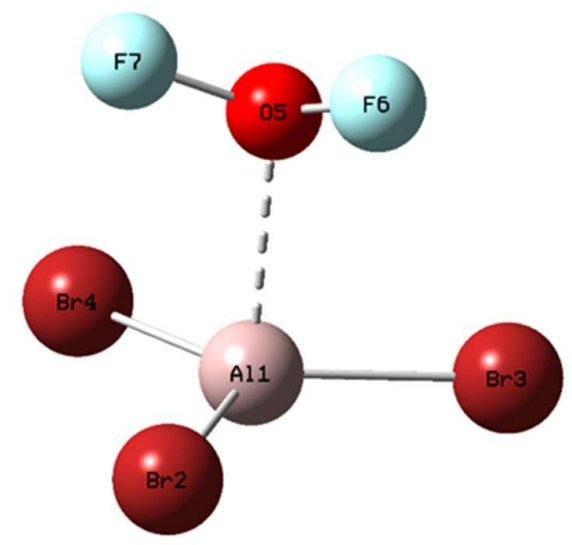

Figure 3. Optimized model of $\mathrm{OF}_{2}$ bound to $\mathrm{AlBr}_{3}$ via the fluoride atom in the charge transfer (CT) complex. The aluminum now adopts a nearly ideal trigonal pyramidal geometry due to the very weak bonding of the oxygen atom to the aluminum atom, with $\angle \mathrm{Br} 3 \mathrm{AlO}=90.38^{\circ}, \angle \mathrm{Br} 2 \mathrm{AlO}=99.52^{\circ}$ and $\angle \mathrm{Br} 4 \mathrm{AlF} 6=90.02^{\circ}$ and $\angle \mathrm{Br} 3 \mathrm{AlF} 6=111.56^{\circ}$, $\angle \mathrm{F} 7 \mathrm{OF} 6=103.96^{\circ}$. The dashed bond between oxygen and aluminum is predicted to be weaker than 0.2 , implying the formation of a weakened CT complex.

The brief thermodynamic analysis for the AlBr3-OF-F shows only slightly different values than for $\mathrm{AlBr}_{3}-\mathrm{F}-\mathrm{OF}$. In fact, the binding process is characterised as slightly endergonic, with a free energy barrier of $+10.975 \mathrm{kcal} / \mathrm{mol}$, which is now consistent with the charge predicted by the NPA and Mulliken population analysis for both the $\mathrm{O}$ and $\mathrm{F}(6)$ atoms, compared to 10.642 $\mathrm{kcal} / \mathrm{mol}$ for the F-bound complex. The small difference in the values of $\Delta G(\mathrm{O}$-bonded) vs. $\Delta G\left(\right.$ F-bonded) (i.e., $\Delta \Delta G_{\text {total }}=0.333 \mathrm{kcal} / \mathrm{mol}$ ) can be easily correlated to the large perturbation of electron density within the OF2 molecule and, more importantly, to very opposite electronic effects discussed later in the text. Moreover, in the case of the O-bonded complex, the stability thermodynamic analyses performed can confirm the endergonic character by revealing a positive total energy barrier of $+5.799 \mathrm{kcal} / \mathrm{mol}$. In the latter case, the charge analysis is in absolute agreement with the basic thermodynamic analyses performed (Table 3 ).

Table 3. NPA, Mulliken and APT charges on M06L/aug-cc-pvDZ level of theory for AlBr3-OF-F complex.

\begin{tabular}{cccccccc}
\hline & $\mathrm{Al}$ & $\mathrm{O}$ & $\mathrm{F}(7)$ & $\mathrm{F}(6)$ & $\mathrm{Br}(2)$ & $\operatorname{Br}(3)$ & $\operatorname{Br}(4)$ \\
\hline $\begin{array}{c}\text { NPA charge } \\
\text { Mulliken }\end{array}$ & 1.222 & 0.162 & -0.053 & -0.053 & -0.429 & -0.425 & -0.424 \\
$\quad \begin{array}{c}\text { charge } \\
\text { APT charge }\end{array}$ & 1.069 & 0.478 & -0.220 & -0.220 & -0.365 & -0.371 & -0.371 \\
\hline
\end{tabular}

Earlier short analyses would have shown that the fluorine atom on $\mathrm{OF}_{2}$ is capable of acting as a nucleophile while binding to a strong Lewis acid such as $\mathrm{AlBr}_{3}$. It can be seen that $\mathrm{OF}_{2}$ is also able to bind to $\mathrm{FeBr}_{3}$, although it is a slightly weaker Lewis acid. However, in order to gain a full insight into $\mathrm{OF}_{2}$ reactivity patterns, we decided to extend the theoretical analysis and perform calculations at an even higher level of theory. Namely, as in previous analyses (see Experimental Section), we treated (monomeric) $\mathrm{OF}_{2}$ at the $\mathrm{MP}_{2}$ level of theory, with a highly 
accurate cc-pV5Z basis set for all atoms, including toluene-solubilizing interactions, to better capture the $\mathrm{OF}_{2}$ interactions in this type of analysis. Based on the hypothesis that the charge pattern of $\mathrm{OF}_{2}$ is as previously discussed, the reasoning could lead to fluorine as a pronucleophilic species. However, the results of the PES analyses have shown somewhat different reactivity pathways, predicting oxygen as the pronucleophilic species rather than fluorine, in stark contrast to the previously predicted reactivity magnitudes for both oxygen and fluorine. At this point, one can become confused with such preliminary assumptions, and therefore an extended analysis of $\mathrm{OF}_{2}$ was deemed necessary, with the precise aim of investigating why oxygen showed better reactivity-modulated assertiveness towards the iron atom than fluorine. Frontier Molecular Orbital (FMO) analysis using eigenvector and Natural Bonding Orbital (NBO) methods [37] was very useful to derive specific details about the interaction pathways. In addition, the theoretical knowledge of the electronic properties of fluorine and oxygen should be correlated with the FMO, eigenvector and NBO analyses to better understand the overall picture of CT complex formation as well as any fluoronium ion formation.

The first thing we observed with the MP2 functionality in the $\mathrm{OF}_{2}$ analysis was that the spontaneity of CT-complex formation remained intact and of almost similar magnitude to AlBr3. Although there are discrepancies between the accuracy of M06L [38], as a meta-GGA DFT method and MP2 [39], as a second-order correlation perturbation method, as well as between the chosen basis sets, we considered the calculated thermodynamic properties on $\mathrm{OF}_{2}$ and $\mathrm{FeBr}_{3}$ and the agreement with the predicted hypotheses was complete, with the same trend in the qualitative interpretation of the results as indicated above. The stabilisation of such CT complexes also remained in the same trend as in the previous analyses, showing an ability in the further analysis of the possible energy deviations and/or predictions about some types of transition states. The knowledge that the oxygen tends to attract electrons to itself, with pronounced negative inductive effect it exerts on the environment (i.e. strong -I effect), leads to a decrease in the electron density over the two fluorine moieties on the molecular map, making the fluorine less reactive at this stage. On the other hand, the fluorine atoms are more electronegative, so the inductive (i.e. via $\sigma$-bonding) withdrawal of electrons from the single oxygen atom will occur in parallel, contradicting the previous statement. $\sigma$-electrons have a larger $\alpha$-coefficient due to their lower mobility over the given molecular map, so inductive contributions in this molecule would be very difficult to predict with such accuracy to perfectly determine which atom would pull more electron density from another! From now on, we turned to another aspect of reactivity that takes into account the gradient of electron motion and should therefore be a better analytical tool in the derivation of reactivity - the resonance contribution. Even if there are no explicit empty (pro-)LUMOs, there are certain electron motions in each molecule that increase the kinetic energy and thus in some cases even the potential energy of a system (molecule). Although this is not fully visible within $\mathrm{OF}_{2}$, both the HOMO -LUMO gap and its shape suggest that there is a significant moment in the unprecedented reactivity of oxygen towards the fluorine atom. Figure 4 shows the orbital shift in $\mathrm{OF}_{2}$, which shows significant electron movement toward the oxygen atom from each fluorine atom, with the electron movement parameter increasing at the fluorine atom rather than the oxygen atom.

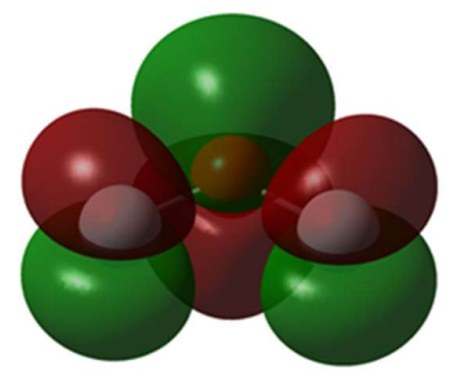

(a)

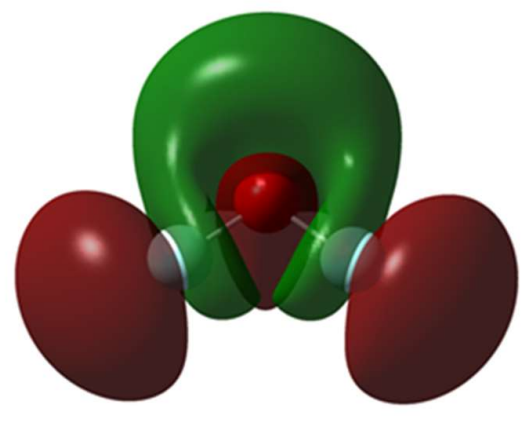

(b)

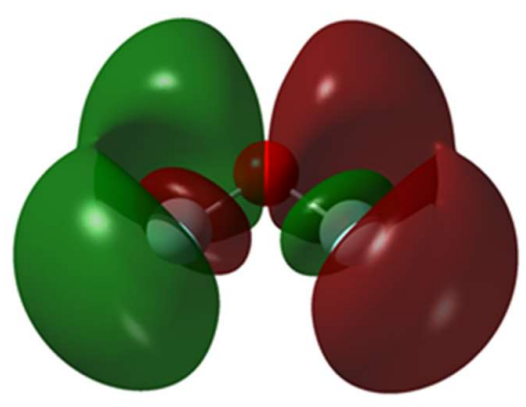

(c) 
Figure 4. $\mathrm{HOMO}(\mathrm{a})$, LUMO (b) and LUMO+1 (c) for OF2 performed at MP2/cc-pV5Z level of theory.

As shown in Figure 4, the HOMO map still shows that there is a significant orbital on oxygen that is fulfilled and pronucleophilic toward electrophiles. On the other hand, to clarify the orbital picture of the LUMO over $\mathrm{OF}_{2}$, there are interacting orbitals on the oxygen leading to a final LUMO located at the top of the oxygen atom, which is probably responsible for the backbonding donation of the fluorine atoms, balancing the electron density from the fluorine atoms to the oxygen atom. To further illustrate the backbonding phenomenon in this case, there is a LUMO+1 map representing the next orbital rearrangement, which is most likely responsible for the (slight) strengthening of the F-O bond. At this point, however, all that needs to be said is that there is a back-bonding that is likely to undergo a dynamic equilibrium state, i.e. electrons flow from one fluorine atom towards oxygen and then from another fluorine atom towards oxygen, pushing electrons towards the first fluorine atom, ultimately leading to the conclusion that the oxygen atom is considered more electron dense than the fluorine atoms themselves. This allows the Laplacian map to very accurately describe where the electron flow was detected on the $\mathrm{OF}_{2}$ molecular map (Figure 5). Reading the Laplacian map, one can clearly see that the electrons are flowing at the edge of the oxygen atom, in the plane where most of the LUMO is located, which can directly mean that there is a backbonding phenomenon that enhances the nucleophilic orbitals on the oxygen.

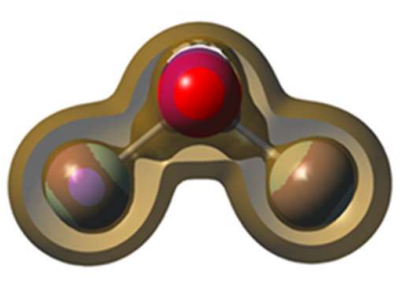

(a)

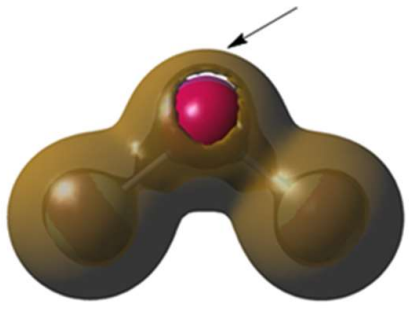

(b)

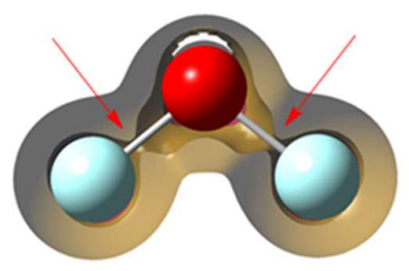

(c)

Figure 5. Z-sliced Laplacian maps (a) and (c) and original Laplacian map (b). The brown surface shows the electron flow as a function of the relative position of the electrons, while the pink surface (below the brown) shows the intrinsic (static) electron density. Surface (b) shows that the pink surface contour on the oxygen is larger than that on the fluorine atoms, and the brown surface (black arrow) denotes electron flow due to backbonding. Red arrows on the surface (c) represent gaps/spaces where electrons can move much slower and therefore form a bond (i.e. form a bonding orbital).

A final additional proof of the superior reactivity of oxygen, along with orbital, Laplacian, and electronic effects, can be provided simply by plotting the electrostatic potential map (MEP/MESP). According to the definition of MEP, the electron density is localized over the entire molecular map of the given compound, with positive parts of the molecular map interacting more intensely with the charge on the surface, resulting in parts where the potential is more positive. Applying this interpretation to the localization of the electron pairs responsible for the reactivity of oxygen, one can clearly see that in the case of oxygen almost the same amount of charge is distributed over both fluorine atoms and perpendicular to the fluorine atoms. At the same time, LUMO and MEP can be fully correlated, resulting in a large positive potential gap between oxygen and fluorine atoms, widely located around the Van der Waals sphere (Fig. 6). 


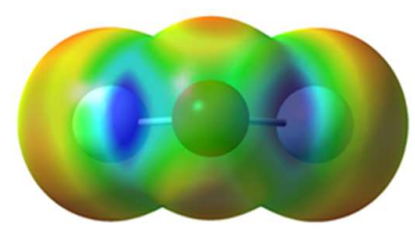

(a)

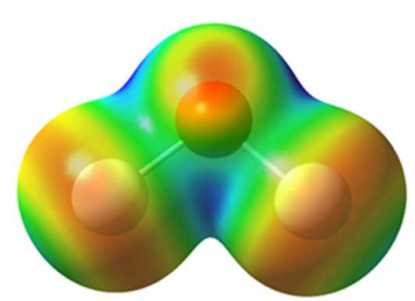

(b)

Figure 6. Molecular Electrostatic Potential (MEP) in plan view (a) and as side view (b). Red areas are values of 0.02-0.03 $\mathrm{AU}$; green areas are values of 0.078 to $0.1 \mathrm{AU}$; blue areas are values of $\geq 0.1 \mathrm{AU}$ (AU = Arbitrary Units).

The $\mathrm{OF}_{2}$ analyses, carried out at the DFT and MP2 levels of theory, aimed to highlight any inconsistencies in the reactivity pathways exerted by $\mathrm{OF}_{2}$, as well as to clarify as much as possible the true nature of $\mathrm{OF}_{2}$ when speaking of electron distribution mechanisms and involvement in charge transfer processes. The extent of the analysis performed on $\mathrm{AlBr}_{3}$ could perhaps tell us something about the reactivity of $\mathrm{OF}_{2}$ with respect to a potential fluorinating agent. At this point, it must be said that the nature of $\mathrm{AlBr}_{3}$ and $\mathrm{FeBr}_{3}$ is similar only in general terms. In order to properly capture the overall picture of the extent of $\mathrm{OF}_{2}$ interaction on $\mathrm{FeBr}_{3}$, we decided to first perform a potential energy analysis and then adopt a fast graphical analysis approach to analyse possible (very) small energy changes that may occur.

In addition to these analyses, the true nature of the binding process must also be evaluated. Using the graphical method, the behaviour of the reacting fluorine or oxygen centre at each theory level can be easily predicted. Figure 7 shows PES of CT complex formation for the system FeBr3-F-OF. The scan was performed at the PM7 semiempirical level of theory due to the complexity of the implementation of the PES algorithm. In fact, the scan was performed at this level of theory because the computational effort to accuracy ratio had been previously evaluated with good correlations, especially to its predecessor, PM6. The curve showed many inconsistencies during the scan, which took the scan out of regular shape. According to the mathematics, such a scan correlates well with polynomial function fitting algorithms, although exponential functions are not excluded. However, this phenomenon was not observed in this scan domain.

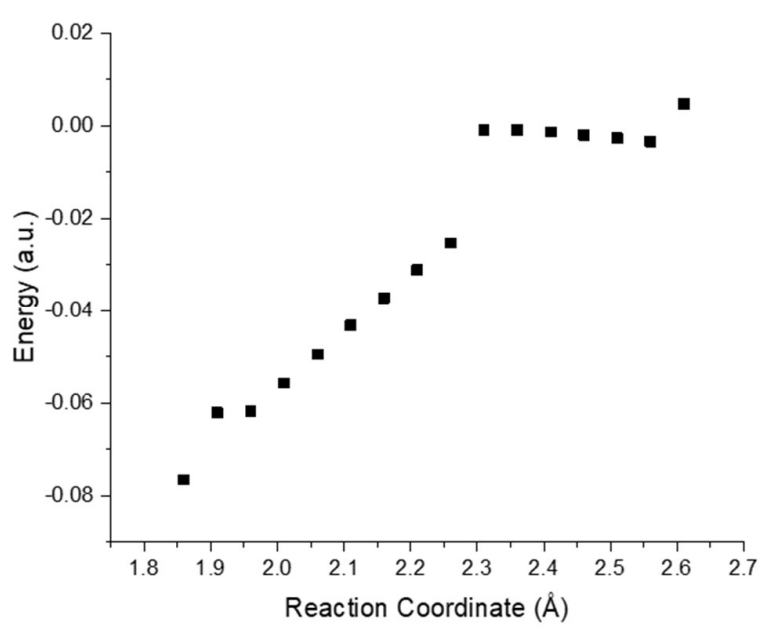

Figure 7. PES for FeBr3-F-OF CT complex. The reaction coordinate was scanned along the Fe-F bond.

The first point of the scan was set at $2.610 \AA$, with a tendency to shorten as a function of time. As the complex moved over time along the given reaction coordinate, which was set as the $\mathrm{Fe}-\mathrm{F}(7)$ bond (Figure 8a), it can be observed that a regular energy drop of about $5 \mathrm{kcal} / \mathrm{mol}$ 
occurred between the first and second scan steps (transition from higher to lower values of the reaction coordinate), causing the energy level to drop to a negative value at this point of the scan. Although a strange energy state was observed for the scan start (a positive energy value is rather discouraging in terms of system stabilization in the scan process), we attributed this rather strange phenomenon to compensation for the weakened (non-covalent) nature of the dispersion interaction between the fluorine atom in $\mathrm{OF}_{2}$ and the Fe atom in $\mathrm{FeBr}_{3}$. The next six scan steps showed an unexpected energy increase, raising the energy of the system from -2.102 to $-0.473 \mathrm{kcal} / \mathrm{mol}$. This unexpected positive energy shift led us to characterize the further evolution of the PES by the simple conclusion that seems to correspond to the analogous $\mathrm{AlBr}_{3}$ system discussed earlier, where it was assumed that a partial positive charge developing on the fluorine atom generally destabilizes the system. Although this type of conclusion fits the results within the PES of FeBr3-F-OF, the further evolution of PES along the reaction coordinate shows that the energy decrease between the seventh and eighth scan steps is quite strong, decreasing the energy of the system up to $15.4 \mathrm{kcal} / \mathrm{mol}$, leading to the idea that this is the first relevant point in this type of transition state evaluation. The next scan steps proceed regularly, with uniform energy decrease, suggesting that the (main) energy barrier has already been crossed. At the fifteenth scan step, the energy gradient was also turned to a positive value, indicating that something is happening in the system at an intrinsic level of the event. When we examined the structures at this point from PES, we noticed an interesting but large change in the system geometry between the fifteenth and sixteenth scan steps. Namely, a large change in bond length in the $\mathrm{O}-\mathrm{Br}(2)$ bond of $\approx 0.08 \AA$, suggesting that the change in energy gradient became too large and therefore induced a rather positive energy change. By examining the structures of PES, produced at these two points, we can confirm this fact by observing a slight increase in the working gradient, which shifts the energy change towards a positive value, strongly indicating the existence of CDR at the fifteenth scan step. Such an existence of CDR points in the course of this PES can be an obstacle in accurately predicting the point where an energy barrier is located on such an energy profile, so the graph PES was modified (Figure $8 \mathrm{~b}$ ) to see if the predicted transition state can be better correlated with the one found by the Berny transition state algorithm [40]. An interesting fact also emerged from the study of such a PES profile of $\mathrm{FeBr}_{3}-$ F-OF. The first point of the scan (i.e., the first scan step) showed an uncanny geometry, perhaps not apparent at first glance, in agreement with the second, with respect to the same $\mathrm{O}-\mathrm{Br}(2)$ bond. In fact, a comparison of the change in bond length, which showed a decrease of $0.35 \AA$ between the first and second scan steps, and a decrease in scan steps from the second to the seventh scan step, which corresponded to 0.01-0.02 $\AA$, gave us an additional idea that the structure in the first scan step can also be considered a different CDR. When CDR is detected at the first point of the energy profile, it is usually referred to as a pseudo CDR structure, since no evidence of a significant geometry change in one direction or the other (from the subsequent next and previous structure in the given PES profile) can be evaluated at this point. 


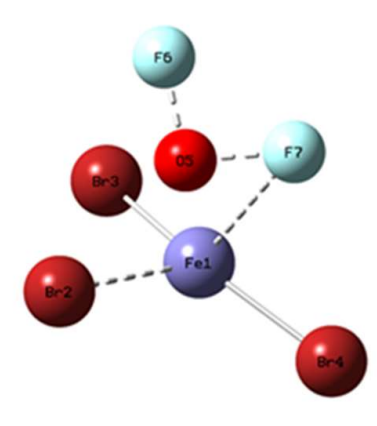

(a)

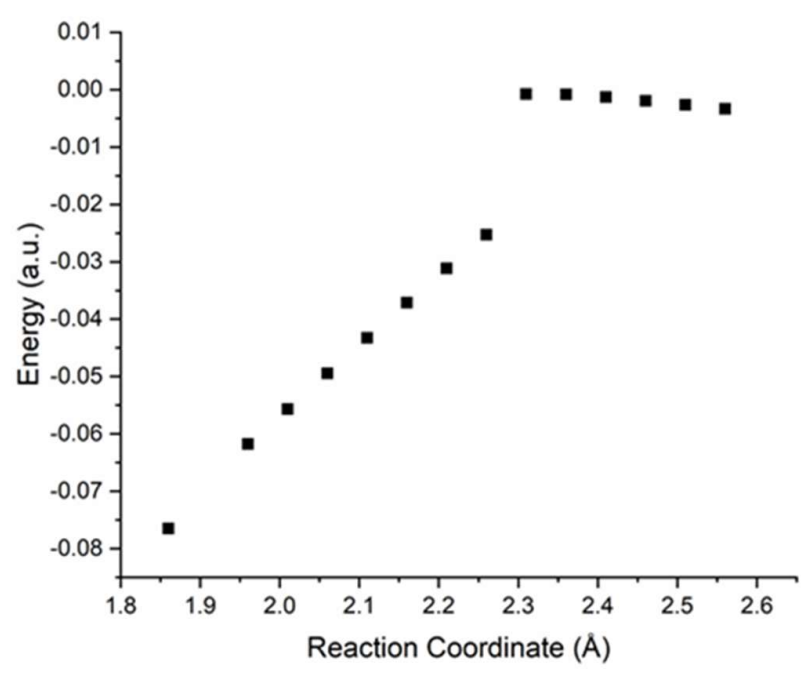

(b)

Figure 8. First scan step for PES of the FeBr3-F-OF system (a) and modified PES profile for the FeBr3-F- OF system, without the structures of the first and fifteenth scan steps, denoted CDR (i.e. CDR -free PES profile) (b). All dashed bonds in (a) are represented by bond order values smaller than 0.7 .

According to the Berny algorithm in the TS characterization for FeBr3-F-OF system, the observed $\mathrm{O}-\mathrm{F}(7)$ bond showed the length of $1.720 \AA$ as the chosen reaction coordinate with converged TS moment. Although a single negative vibration was found for this system in the characterization of TS, this single negative vibration corresponds to the mutual wedging of OF and $\mathrm{FeBr}_{3} \mathrm{~F}$ fragments in space, ruling out the negative frequency as responsible for defining the TS moment. Interestingly, a strong stretching vibration was assigned for the $\mathrm{O}-\mathrm{F}(7)$ bond at 764 $\mathrm{cm}-1$, which is a rather unusual feature of a transition state found in the positive value region of the predicted IR spectrum. This characterization led to the fact that no "real" TS can be assigned to this system, introducing the possibility of the existence of another type of transition state. Considering the nature of the interaction that can occur between these two molecules $\left(\mathrm{FeBr}_{3}\right.$ and $\left.\mathrm{OF}_{2}\right)$ in this system, it is most likely to define such an energy barrier as a pretransition state (preTS). Analyzing the thermochemistry of the given TS, it can be clearly stated that the process of binding fluorine to the $\mathrm{Fe}$ atom in $\mathrm{FeBr}_{3}$ is exergonic, with the value of 19.084 $\mathrm{kcal} / \mathrm{mol}$, which was further supported with the total stabilization energy, with the value of $65.542 \mathrm{kcal} / \mathrm{mol}$. The results shown here are a strong indication of the spontaneity of the fluorine bond in the CT complex. Using the SOD analysis showed results outside the range studied by PES. In our opinion, such a small value for preTS predicted for this CT complex is indeed unusual and is at the limit of Fe-F binding in $\mathrm{FeF}_{3}$ [41]. This fact changes the course of the SOD analysis, which shows an obvious error of more than $0.4 \AA$ in the bond length. Figure $9 \mathrm{a}$ and $9 \mathrm{~b}$ show the plot of the SOD analysis, where the predicted preTS values for this type of bond were obtained at $2.187 \AA$ (for the regular PES profile) and at $2.139 \AA$ (for the CDR-free PES profile). The deviation of the obtained values from the predicted preTS values is summed up in Table 4 . and can be characterized as high, with respect to the degree of accuracy we are looking for. 


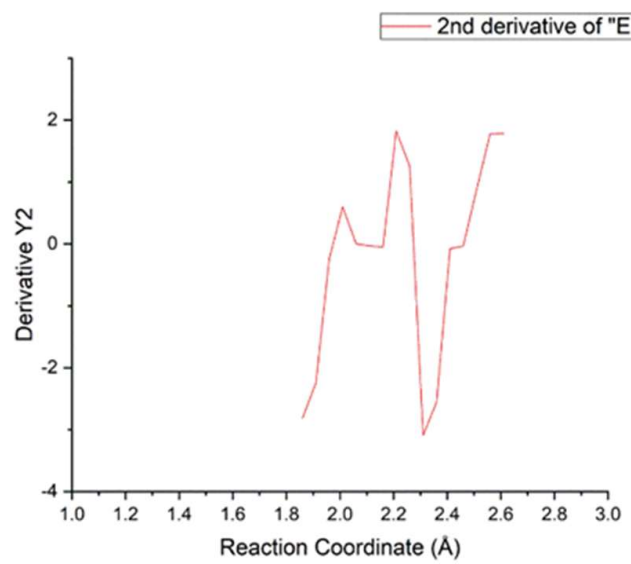

(a)

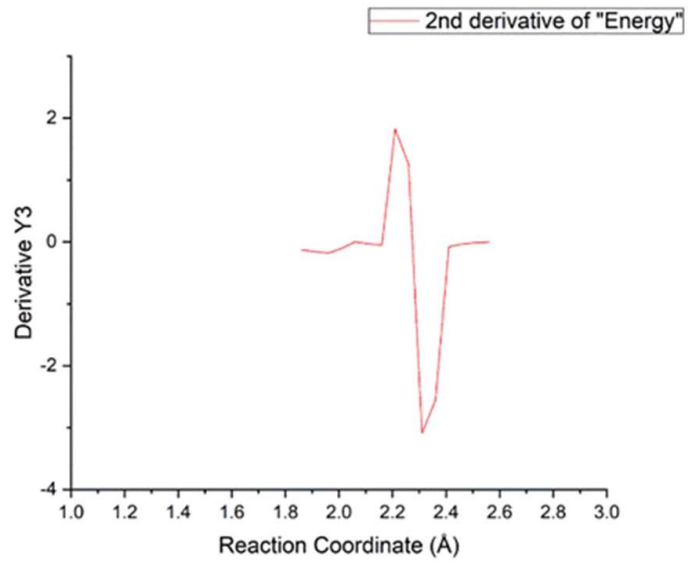

(b)

Figure 9. SOD plot for original PES scanning Fe-F(7) bond (a) and SOD plot for CDR-free PES profile scanning Fe-F(7) bond (b).

Table 4. Pre-transition state value obtained with the Berny algorithm at the PM7 semiempirical level of theory compared to interpolated values obtained by SOD analysis from analogous PES profiles.

\begin{tabular}{cccccc}
\hline & $\begin{array}{c}\text { Berny algorithm } \\
(\AA)\end{array}$ & $\begin{array}{c}\text { SOD for complete PES } \\
(\AA)\end{array}$ & $\begin{array}{c}\text { SOD for CDR-free PES } \\
(\AA)\end{array}$ & $\begin{array}{c}\text { Absolute error } \\
(\AA)\end{array}$ & $\begin{array}{c}\text { Relative error } \\
(\%)^{*}\end{array}$ \\
\hline $\begin{array}{c}\text { O-F(7) } \\
\text { bond }\end{array}$ & 1.720 & & & 0.467 (regular) & 27.15 (regular) \\
length & & 2.187 & 2.139 & $0.419(\mathrm{CDR}-$ & $\begin{array}{c}24.36(\mathrm{CDR}- \\
\text { free) }\end{array}$ \\
\hline
\end{tabular}

* Relative error determination principle is described in Experimental Section

On the other hand, after the formation of the CT- complex, in a temporally subsequent phase, the fluoronium ion is formed as an electrophile immediately before the nucleophilic attack by the aromatic compound. This slight difference between the formation of the $\pi$ complex in the early transition state of the EAS and the formation of the fluoronium ion can also be theoretically evaluated by a 1D potential energy scan of the independent TS for the aromatic $\pi$-bond and the formation of the fluoronium ion in the F-bonded FeBr3-F-OF complex. PES profile of fluoronium ion formation in the F-binding is shown in Figure 10.

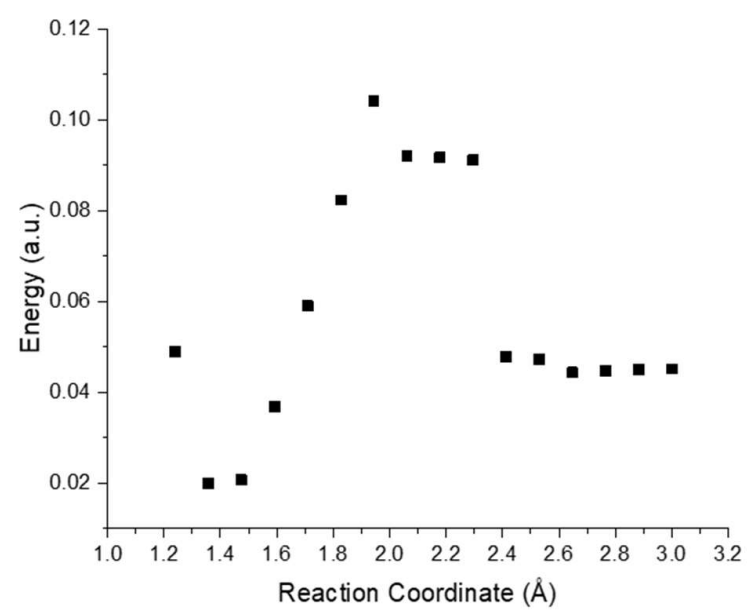

Figure 10. PES for $\mathrm{FeBr}_{3}-\mathrm{F}-\mathrm{OF}$ fluoronium ion complex. The reaction coordinate was scanned along the O-F(6) bond. 
Analyzing the energy profile within PES for fluoronium ion complex formation, we see that the formation of the fluoronium ion via the F-bonded complex is nearly thermoneutral. At first glance, the profile of PES does not tell us much except that there is an increased frequency of change in the energy gradient, which means that the working gradient also changes relatively frequently. Therefore, we could locate at least two CDR points: at the first and twelfth scan steps (starting from lower to higher values of the reaction coordinate). Using the SOD analysis (Figure 11) without excluding the CDR points told us that the possible fluoronium ion transition state could be at $2.316 \AA$. Unfortunately, we have not yet been able to converge to the optimization of such a transition state using the Berny algorithm, so there is no relevant reference data for comparison. Our conjecture for the lack of convergence for the transition state of the fluoronium ion across the F-bonded complex is that an energy increase occurred in the species formed during the transition state - the oxyfluoride cation $\left(\mathrm{F}-\mathrm{O}^{+}\right.$ion $)$- which could not be sufficiently stabilized even via the pseudoresonance pathway from fluoride backbonding electron donation. Therefore, based on the computational convergence criterion, we can rule out that the fluoride pathway binds to $\mathrm{FeBr}_{3}$, leaving the possibility that oxygen is the relevant species within $\mathrm{OF}_{2}$ where the binding center could be located.

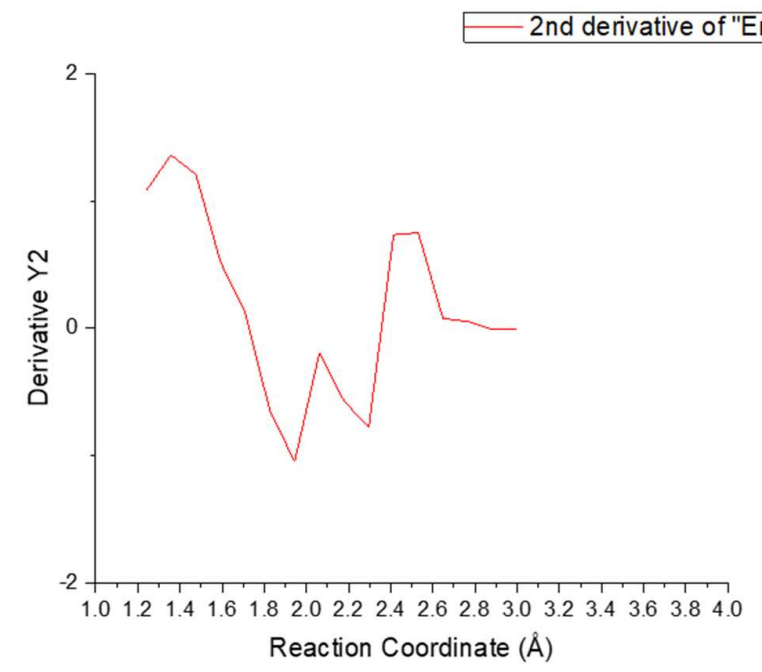

Figure 11. SOD Plot for the formation of fluoronium ions for the FeBr3-F-OF CT-complex when the O-F(6) bond was chosen as the reaction coordinate.

The case of the O-bonded FeBr3- OF-F complex can be described as more straightforward with one exception. The PES profile for this binding pathway can be characterized in a smoother manner, starting at $2.730 \AA$. As can be seen from Figure $12 b$, the transition from higher to lower values of the reaction coordinate results in a slight decrease in energy, which is favored only for the CT -complexation process. The trend of energy decrease continues until the ninth scan step, where we observe a larger perturbation of the energy gradient, leading us to believe that the ninth point can be characterized as the first observable CDR point at $-0.1616 \mathrm{kcal} / \mathrm{mol}$. In agreement with the trend of energy decrease, the energy values up to the eighth sampling step also had a positive sign and ended with the eighth point $\left(\mathrm{E}_{8}=0.2471 \mathrm{kcal} / \mathrm{mol}\right)$. However, at the transition from the ninth to the tenth scan step, a different value appears which, although not different in energy gradient from the ninth point, has an additional value that lowers the energy of the system $\left(\mathrm{E}_{10}=-0.4241 \mathrm{kcal} / \mathrm{mol}\right)$. This case does not occur so often when two CDRs are identified in succession, so our consideration was that both could affect the accuracy of the preTS estimate by the graphical method. The next three scan steps proceed via an energy rise, which could be characterized as a kind of strange behavior of a PES profile in the third quadrant of the profile, although one would expect the value of the energy to decrease by default. The opposite case of the energy increase could have led us to conclude that some, perhaps unusual, reactivity occurs during the process. Simulating PES profiles at all semiempirical levels, 
including the most powerful PM7 functional to date, leads to greater error production due to the complexity of the PES algorithms themselves. We have observed such a trend in this example, where it can be seen that the last three values (fourteenth to sixteenth scan step) have much lower energy than the rest of the plot. In examining the last three scan steps, we observed something that, to our knowledge, has not been reported in the literature - the formation of tribromooxyfluoroiron(V) fluoride (Figure 13a) [42]. Such a transition from iron(III) to iron(V) can only be described for inorganic species, and that is by passing iron(III) compounds through the current of a very strong oxidizing agent. $\mathrm{OF}_{2}$ is also a strong oxidant, but what was strange for us is the character of the $\mathrm{Fe}-\mathrm{F}(3)$ bond, as well as the cleavage of the $\mathrm{O}-\mathrm{F}(3)$ bond during the binding process. Our analyzes at the DFT theory level were not shown in this manuscript, but they were performed on these compounds to investigate the thermochemistry and stability of such processes and to inspect the nature of the given $\mathrm{Fe}-\mathrm{F}(3)$ bond. At the final stage, our opinion here is that a significant energy decrease has occurred due to the stabilizing effect that the ferrate ion has on the adjacent fluoronium ion that is subsequently formed.

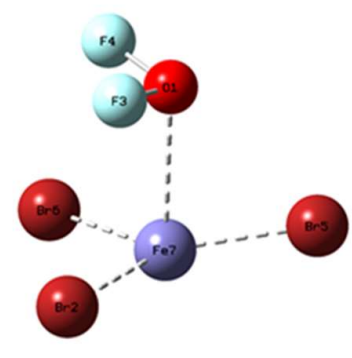

(a)

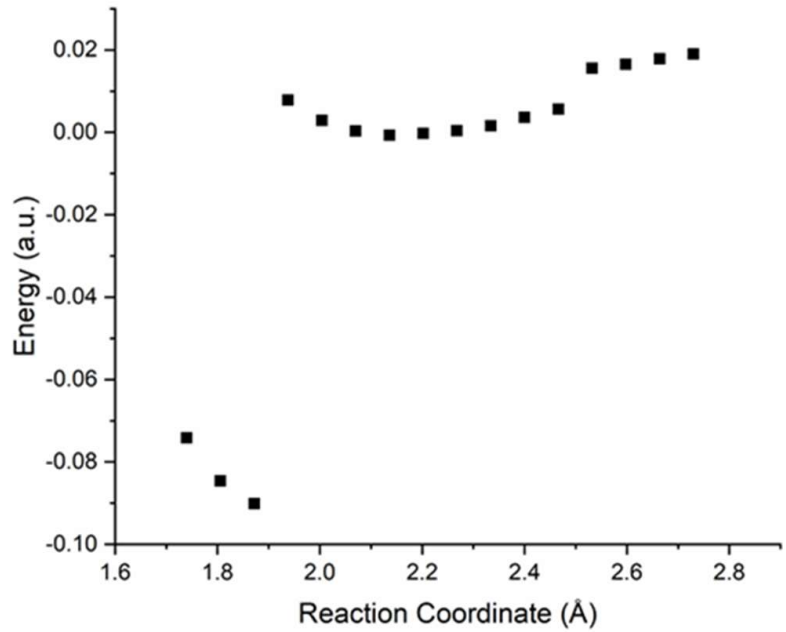

(b)

Figure 12. First scanning step for PES of FeBr3- OF-F system (a) and PES profile for oxygen binding during the formation of the CT complex (b).

When analyzing the potential preTS in this O-bonded CT complex, the Berny algorithm showed an Fe-O bond value of $2.167 \AA$. Again, as in the case of the F-bonded complex, a single negative vibration was found at $20.3 \mathrm{~cm}^{-1}$, although this is not very informative as this is not the frequency corresponding to the pretransition state being sought. Interestingly, as in the previous analogous example with the F-linked complex, an oscillation was found at $420.3 \mathrm{~cm}^{-1}$, which best corresponds to the sought pretransition state. Contrary to our assumptions, this frequency was one with attenuated intensity, reflecting a combined bending (between two fluorine atoms) and stretching (Fe-O bond) vibration. Thermochemical analyzes revealed an exergonic behavior with binding favored by $19.637 \mathrm{kcal} / \mathrm{mol}$. To our surprise, the total stabilization energy in this CT complex formation was also $19.423 \mathrm{kcal} / \mathrm{mol}$ in favor of spontaneity. With regard to the discussion of energy levels, we can say that these values for Obonded CT complex formation are in good agreement with experimentally obtained values and fit into the general range of 0.5 to $21 \mathrm{kcal} / \mathrm{mol}$ [43]. Moreover, the appearance of a positive frequency characterized as preTS again suggests that such a non-covalent interaction cannot be classified as a true transition state, but can be found in the vicinity of the true transition state (in which case it would possibly be $\pi$-complex formation, but we would be very cautious about making such a statement). Moreover, the geometry of the O-linked CT complex suggests that the trigonal pyramidal symmetry could be an encouraging element in the analysis of further halonium ions to this complex. 
Table 5. Pretransition state value determined by Berny algorithm at PM7 semiempirical level of theory compared to the interpolated values obtained via the SOD analysis from analogous PES profiles (Figure 14).

\begin{tabular}{|c|c|c|c|c|c|}
\hline & $\begin{array}{c}\text { Berny algorithm } \\
(\AA)\end{array}$ & $\begin{array}{l}\text { SOD for complete PES } \\
\qquad(\AA)\end{array}$ & $\begin{array}{l}\text { SOD for CDR-free PES } \\
\qquad(\AA)\end{array}$ & $\begin{array}{l}\text { Absolute error } \\
(\AA ̊)\end{array}$ & $\begin{array}{l}\text { Relative error } \\
\qquad(\%)\end{array}$ \\
\hline $\begin{array}{l}\mathrm{Fe}-\mathrm{O} \\
\text { bond } \\
\text { length }\end{array}$ & 2.167 & 2.357 & 2.278 & $\begin{array}{c}0.190 \text { (regular) } \\
0.111 \text { (CDR- } \\
\text { free) }\end{array}$ & $\begin{array}{c}8.77 \text { (regular) } \\
5.12 \text { (CDR-free) }\end{array}$ \\
\hline
\end{tabular}

In Table 5, it can be clearly seen that the value from the CDR-free PES profile for the Obound CT-complex is close to the range of analytical error, which is undoubtedly due to the oxygen binding process. Also, the regular PES profile can be used to accurately explain the preTS determination in this type of binding.

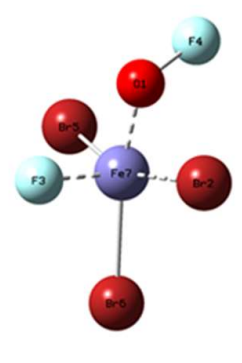

(a)

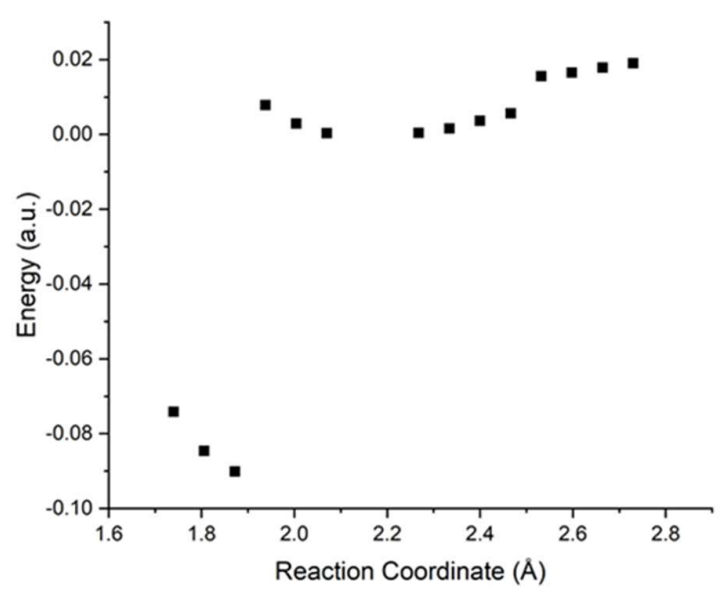

(b)

Figure 13. Final scan step for PES of FeBr3-OF-F system - tribromooxyfluoroiron(V) fluoride (a) and CDR-free PES profile for oxygen binding in the formation of the CT complex (b).

The identification of the fluoronium ion in the PES -profile, when the O-linked CTcomplex was analyzed, was actually similar to the $\mathrm{FeBr}_{3}-\mathrm{OF}-\mathrm{F}$ system discussed in the previous sections. The only difference in the formation of the CT-complex in the FeBr3-OF-F -system was that the PES profile gave a better flux function and perhaps more distinct CDRs. Indeed, the first and second scan steps differed in a way where the energy gradient increased with increasing energy of the second scan step structure. Interestingly, we observed no significant changes in the position gradient and the second step structure differed only slightly from the first step structure in terms of geometry. In contrast, the structures of the third and subsequent scan steps showed significantly larger position gradient changes, which were reflected in smaller energy gradient changes. From the fourth scan step to the end of the PES profile, we did not show any irregular or strange behavior in terms of energy or position gradient changes, only the curvilinear flow function in the ninth scan step towards more positive stabilization energy values converged smoothly in the sixteenth scan step. Nevertheless, we were able to identify at least two CDRs at different points in the PES profile that we could easily exclude from the profile to accurately predict the now given pseudo-transition state of fluoronium ion formation. Therefore, we considered the first, fourth, and ninth scan steps. Despite the complete identification and characterization of the CDRs, the SOD analysis of the regular PES profile for this O-linked fluoronium ion complex yielded a rather strange result, as the function crossed at $1.690 \AA$, which is almost $0.9 \AA$ different from the pseudo-transition state (pseudoTS) found, which is reported to be $2.512 \AA$. A practical solution to this discrepancy emerged upon further 
investigation of the SOD plot - the use of the extrapolation tool. Extrapolating the last part of the function, which was mostly linear in the last part, gave us the idea to extrapolate it linearly. The results are shown in Table 6 and Figure 15.

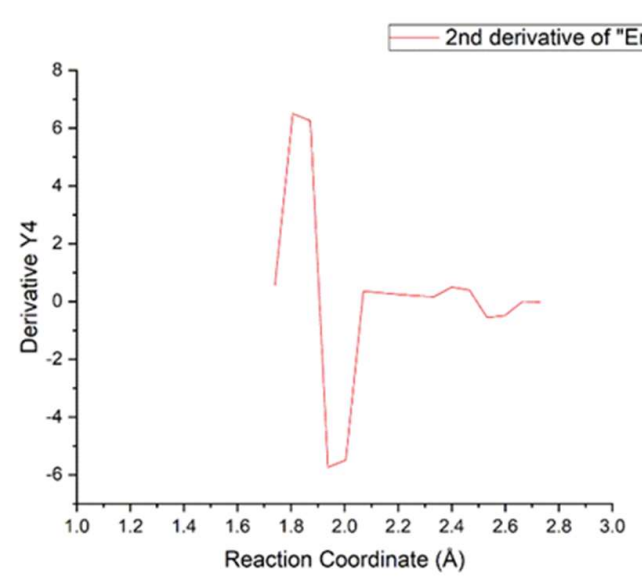

(a)

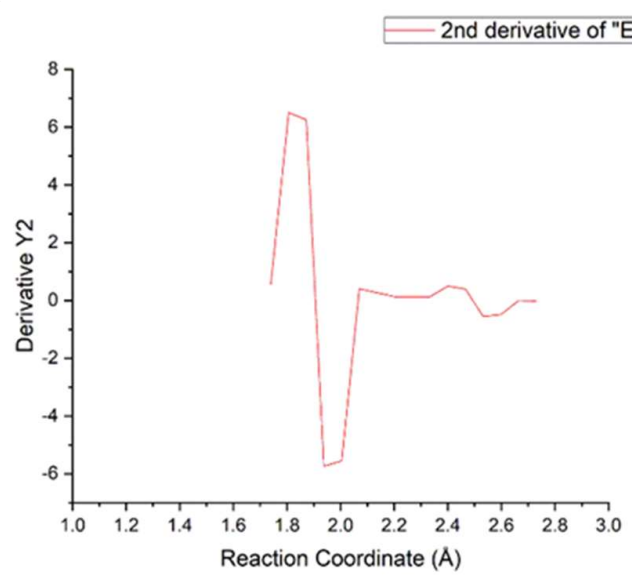

(b)

Figure 14. SOD Plot for the formation of the fluoronium ion complex for FeBr3-OF-F-complex when the Fe-O bond was chosen as the reaction coordinate.

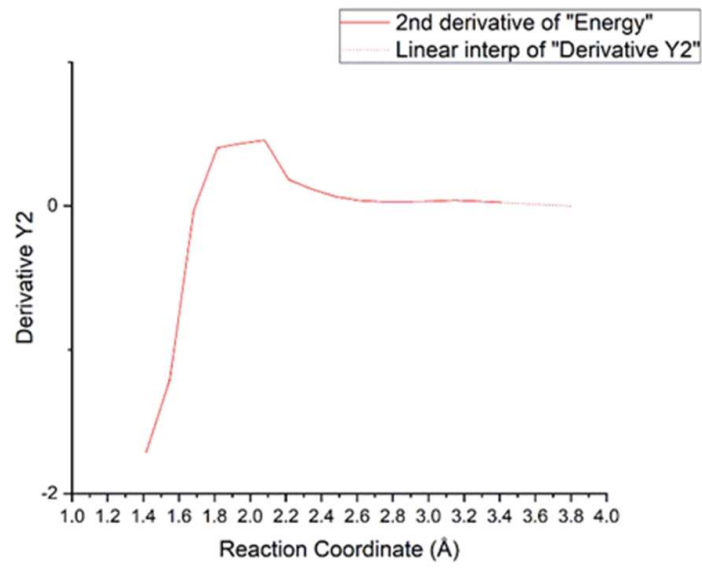

(a)

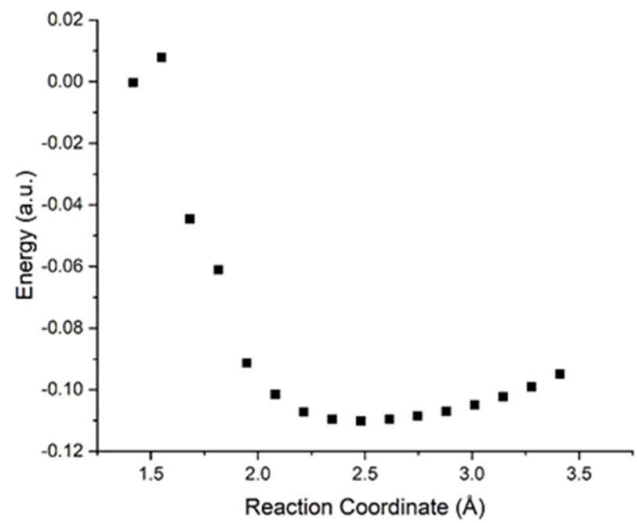

(b)

Figure 15. SOD Plot for the pseudoTS determination of fluoronium ions when the reaction coordinate is $\mathrm{O}-\mathrm{F}(3)$ (a) and regular PES profile for the FeBr3-OF-F complex when fluoronium ions are detected along the O-F(3) bond.

Table 6. Value of the pre-transition state obtained with the Berny algorithm at the PM7 semiempirical theory level compared to the interpolated values obtained via SOD analysis from analogous PES profiles (Figure 14).

$\begin{array}{lllll}\text { Berny algorithm } & \text { SOD for complete PES } & \text { SOD for CDR-free PES } & \text { Absolute error } & \text { Relative error }\end{array}$

$(\AA)$

$(\AA)$

$(\AA)$

$(\AA)$

(\%)

\begin{tabular}{|c|c|c|c|c|c|}
\hline \multicolumn{6}{|l|}{$\mathrm{O}-\mathrm{F}(3)$} \\
\hline bond & 2.512 & 2.733 & $\mathrm{ND}^{*}$ & 0.221 (regular) & 8.80 (regular) \\
\hline length & & & & & \\
\hline
\end{tabular}

${ }^{*} \mathrm{ND}=$ Not Defined

Overall, the thermodynamic analysis of the fluoronium ion transition state shows a rather exergonic behavior, promoting the spontaneous reaction of fluoronium ion transfer from 
oxygen to iron, with a given energy barrier of $19.814 \mathrm{kcal} / \mathrm{mol}$. In this case, this value of $\Delta G$ could be considered reasonable since another step of charge transfer has indeed occurred in this

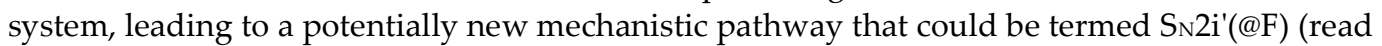
as bimolecular pseudonucleophilic intrasubstitution via fluorine), which was the main characterization thread in the analyzes of both the CT oxygen binding and fluorine ion formation complexes with respect to the $\mathrm{FeBr}_{3}-\mathrm{OF}-\mathrm{F}$ system. Anyway, more on the mechanistic study can be found in Supplementary Material. With regard to the reactivity of $\mathrm{OF}_{2}$ with $\mathrm{FeBr}_{3}$, it is only worth mentioning that the total stabilization energy of such a complex for the formation of fluoronium ions shows a rather high stability for a complex system, with an energy barrier of $-73.147 \mathrm{kcal} / \mathrm{mol}$, for which it can be said to exceed the stability barrier much more than assumed, strongly favoring the formation of fluoronium ions via the oxygen-bonding mechanism. To further support this fact, a IR -analysis revealed a rather unusual circumstance: quenching of the fluoronium ion could be evaluated by a very strong Fe-F stretching band at $844.2 \mathrm{~cm}-1$ with an absent O-F stretching band, which might suggest that the low stability barrier of $-73.147 \mathrm{kcal} / \mathrm{mol}$ could be responsible for a rapid transfer of the fluoronium ion from oxygen to iron, reacting with the ion in an ion-pair fashion and producing a strong non-covalent interaction.

\subsubsection{Chlorine bound to $\mathrm{FeBr}_{3}$}

Chlorine is a yellowish, pungent-smelling gas under standard conditions, considered to be a strong oxidizing agent, and its standard electrode potential is slightly less than $1.5 \mathrm{~V}\left(E^{\circ}=\right.$ $1.360 \mathrm{~V}$, eq. 29) [35]. The chlorine molecule is nonpolar and covalent, which means that the two chlorine atoms are very difficult to polarize unless an external catalytic species is present. In terms of reactivity, chlorine can react with highly polar substances, which partly explains its property as an oxidizing agent that polarizes itself and allows further interactions of the two chlorine atoms. In water, chlorine can react via the activation mechanism of proton transfer to form $\mathrm{HCl}$ and $\mathrm{HClO}$ (hypochlorous acid), with an equilibrium/acid dissociation constant of $\approx$ $10^{-9}$ (eq. 30). Moreover, chlorine acts as an even stronger oxidizing agent and increases the standard electrode potential up to $0.06 \mathrm{~V}\left(E^{\circ}=1.396 \mathrm{~V}\right.$, eq. 31) [35]. With Lewis acids, such as $\mathrm{FeBr}_{3}$, the chlorine molecule interacts via one of its HOMO p orbitals and forms a CT complex.

$$
\begin{aligned}
& \mathrm{Cl}_{2(\mathrm{~g})}+2 e^{-} \rightleftharpoons 2 \mathrm{Cl}^{-} \\
& 2 \mathrm{HClO}+2 \mathrm{H}_{(a q)}^{+}+2 e^{-} \rightleftharpoons \mathrm{Cl}_{2}+2 \mathrm{H}_{2} \mathrm{O} \\
& \mathrm{Cl}_{2(a q)}+2 e^{-} \rightleftharpoons 2 \mathrm{Cl}_{(a q)}^{-}
\end{aligned}
$$

PES profile for the binding of chlorine to $\mathrm{FeBr}_{3}$ is much less complex than for the previous fluorine. The straight energy gradients are almost the same in the first half of the steps needed to build the energy profile. A very smooth and linear energy drop occurs during the first five scan steps, leading to a steep energy drop at the sixth scan step, with an eventual larger energy increase in subsequent steps. The resulting flow function fits the exponential trend line for the second part of the PES profile. The first part of the profile fits linearly, which theoretically would allow easy determination of preTS. A possible explanation for the larger energy gradient in the final steps can be attributed to the same observed phenomenon - the formation of iron(V) species now characterized for the chlorine complex - tribromochloroiron (V) chloride.

Thermochemical analysis of the predicted preTS using the Berny algorithm gave a value of $\mathrm{Fe}-\mathrm{Cl}(5)$ bond length of $2.501 \AA$. Similar to $\mathrm{OF}_{2}$, a single negative frequency was observed in the converged TS structure, but similar to $\mathrm{OF}_{2}$, only one positive frequency, located at $175.2 \mathrm{~cm}^{-}$ 1 , is responsible for the straight-line $\mathrm{Fe}-\mathrm{Cl}(5)$ stretching, which is characterized by a relatively weak intensity of the vibrational shift. With respect to the spontaneity of preTS formation, it should be emphasized that the energy barrier for the chlorine binding process is rather below the barrier with a value of $-23.422 \mathrm{kcal} / \mathrm{mol}$ for both fluorine-bound complexes. On the other hand, the barrier of the chlorine complex in terms of overall stabilization of the CT complex is only $\approx 0.2 \mathrm{kcal} / \mathrm{mol}$ lower than the barrier for fluorine over oxygen-bound complexes, which is 
relevant to the discussion of the energy gap for chlorine-bound complexes and suggests that there are also potentially higher values for the HOMO -LUMO gap, implying that chlorine, although nonpolar, can act like a polarized species over $\mathrm{OF}_{2}$. Moreover, the SOD analysis of the $\mathrm{FeBr}_{3}-\mathrm{Cl}_{2}$ complex gave a preTS value of $2.577 \AA$, which is in perfect agreement with the value calculated at the semiempirical PM7 level (Table 7). Since no CDR is observed within this PES profile, the direct determination of the preTS value is fully supported by the SOD analysis (Figure 16). Moreover, the value of 3.04\% relative error according to the SOD analysis shows for the first time the accuracy of a semiempirical method at the analytical level, which opens new possibilities and horizons in the analysis of even such difficult prediction tasks, as PES, with semiempirical functional(s).

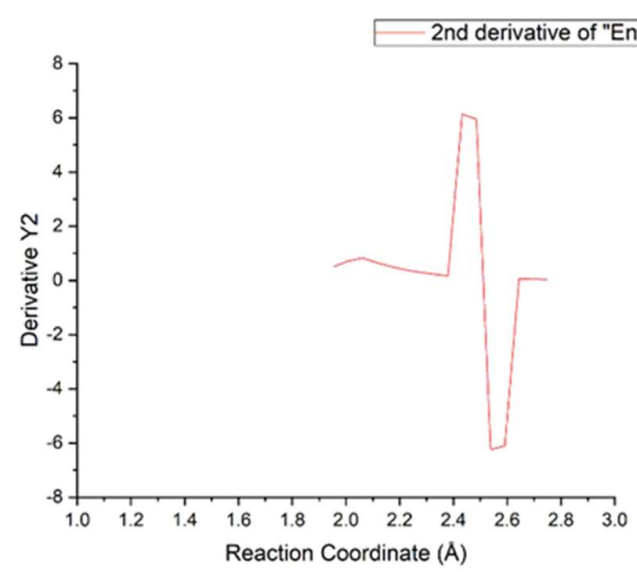

(a)

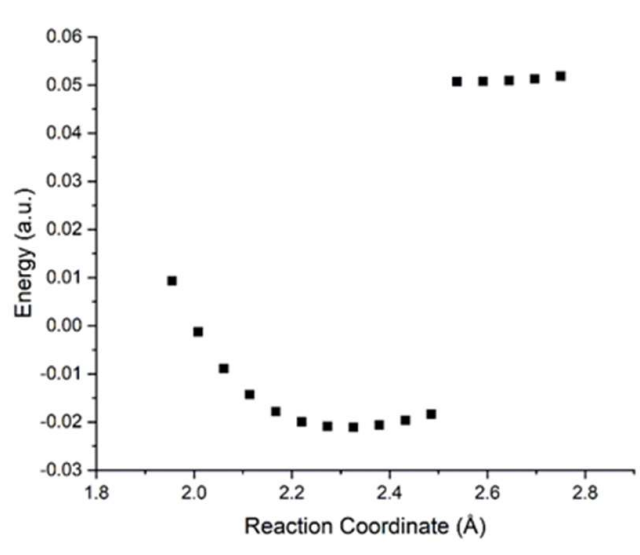

(b)

Figure 16. SOD plot for $\mathrm{FeBr}_{3}-\mathrm{Cl}_{2} \mathrm{CT}$ complex (a) and regular PES profile for $\mathrm{FeBr}_{3}-\mathrm{Cl}_{2} \mathrm{CT}$ complex, with $\mathrm{Fe}-\mathrm{Cl}(5)$ bond chosen as reaction coordinate $(b)$.

Table 7. Value of the pre-transition state determined with the Berny algorithm at the PM7 semiempirical level of theory and the value obtained by SOD analysis for the transition state of the chloronium ion in the $\mathrm{FeBr}_{3}-\mathrm{Cl}_{2} \mathrm{Complex}$.

\begin{tabular}{|c|c|c|c|c|c|}
\hline & $\begin{array}{c}\text { Berny algorithm } \\
(\AA)\end{array}$ & $\begin{array}{l}\text { SOD for complete PES } \\
\qquad(\AA)\end{array}$ & $\begin{array}{l}\text { SOD for CDR-free PES } \\
\qquad(\AA)\end{array}$ & $\begin{array}{l}\text { Absolute error } \\
\qquad(\AA)\end{array}$ & $\begin{array}{c}\text { Relative error } \\
(\%)\end{array}$ \\
\hline \multicolumn{6}{|c|}{$\mathrm{Fe}-\mathrm{Cl}(5)$} \\
\hline bond & 2.501 & 2.577 & $\mathrm{ND}^{*}$ & 0.076 (regular) & 3.04 (regular) \\
\hline length & & & & & \\
\hline
\end{tabular}

${ }^{*} \mathrm{ND}=$ Not Defined

PES profile to identify chloronium ions in the $\mathrm{FeBr}_{3}-\mathrm{Cl}_{2} \mathrm{CT}$ complex is quite straightforward based on previous analyses. Nevertheless, simple assumptions based on previous $\mathrm{Fe}-\mathrm{Cl}$ scans, even with a changed reaction coordinate involving $\mathrm{Cl}(5)-\mathrm{Cl}(6)$ bonding, also yield fairly straightforward PES profiles. According to one such PES plot (Figure 18), a significant energy drop was identified between the third and fourth scan steps and also between the tenth and eleventh scan steps, with a larger energy difference between the third and fourth scan steps. The rationale for this result lies in the fact that the PES algorithm predicted the previous dissociation of the $\mathrm{Cl}(5)-\mathrm{Cl}(6)$ bond in the second scan step (Figure 17), with a significant change in the position gradient in the third scan step. The entire complex clearly transitions from a planar to a trigonal pyramid to a trigonal bipyramidal geometry. The change in geometry is the main factor causing the two most important changes in the energy decay. In the tenth scan step, PES still consists of very weakened $\mathrm{Fe}-\mathrm{Cl}(6)$ as strongly nonbonding, even for noncovalent interactions. In the eleventh scan step this bonding is present and the geometry changes towards trigonal bipyramidal. 
Thermochemical analysis revealed interesting facts. Indeed, pseudoTS was characterized as exergonic at $21.587 \mathrm{kcal} / \mathrm{mol}$, which is a slightly lower energy barrier than for the fluorinebound complexes but slightly higher than for preTS for the chlorine-binding process. This implies that the chloronium ion formation process is rather balanced, which is not so easily seen when considering the stabilization energy barrier, which reaches $-47.847 \mathrm{kcal} / \mathrm{mol}$. This would imply that it is more stable than the chlorine-bonding process, considering that the PES algorithm again predicts the formation of iron(V)-chlorinated species in the final steps, which should be an ion-pair-like structure that is additionally stabilized, lowering the energy barrier. In this case, no single negative frequencies are registered, but uniformly - the frequency at 536.46 $\mathrm{cm}^{-1}$ characterizes the strongest peak with the $\mathrm{Fe}-\mathrm{Cl}(6)$ bond stretching vibration, further justifying the idea that states near TS are characterized with positive values only, albeit stronger than the rest of the calculated ones!

The Berny algorithm predicted $3.012 \AA$ pseudoTS. On the other hand, the analysis of SOD gave values of $2.446 \AA$, which is much less than predicted. Considering that the chloronium ion was predicted to be in the form of an ion pair, this difference should be viewed with further caution, and we believe it is prudent not to make cautious judgments about such large differences in length. Table 8 summarizes the details of the bond length approximations of the two methods. The relative determination error between the two methods of 18.79 encourages us to suggest the SOD method in further evaluations, both in terms of the functional chosen and the complexity of the system(s) analyzed.

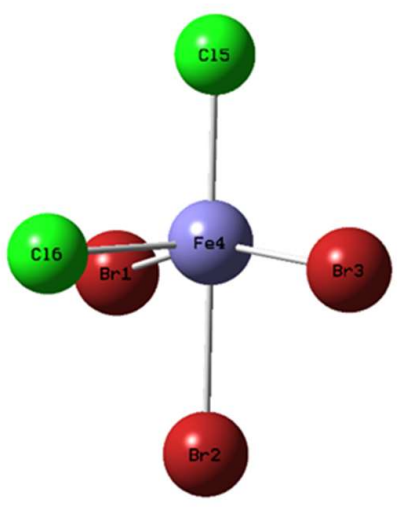

Figure 17. Trigonal bipyramidal geometry approximation for tribromochloroiron(V) chloride, a compound that is supposed to quench and stabilize the chloronium ion.

Table 8. Pseudo-transition state value determined by the Berny algorithm at PM7 semiempirical level of theory and that obtained by SOD analysis for the chloronium ion transition state in the $\mathrm{FeBr} 3-\mathrm{Cl}-\mathrm{Cl}$ complex.

\begin{tabular}{cccccc}
\hline & $\begin{array}{c}\text { Berny algorithm } \\
(\AA)\end{array}$ & $\begin{array}{c}\text { SOD for complete PES } \\
(\AA)\end{array}$ & $\begin{array}{c}\text { SOD for CDR-free PES } \\
(\AA)\end{array}$ & $\begin{array}{c}\text { Absolute error } \\
(\AA)\end{array}$ & Relative error $(\%)$ \\
\hline $\begin{array}{c}\mathrm{Cl}(5)-\mathrm{Cl}(6) \\
\text { bond } \\
\text { length }\end{array}$ & 3.012 & 2.446 & $\mathrm{ND}^{*}$ & 0.566 (regular) & 18.79 (regular) \\
\hline
\end{tabular}

${ }^{*}$ ND = Not Defined 


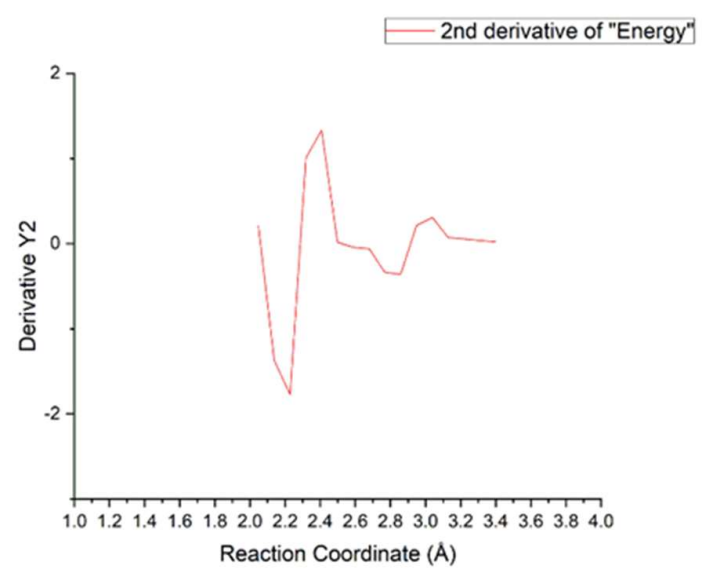

(a)

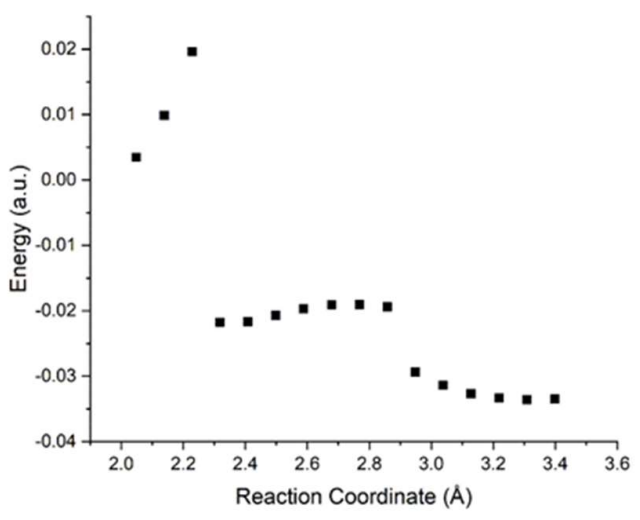

(b)

Figure 18. SOD plot for FeBr3-Cl-Cl CT complex (a) and regular PES profile for FeBr3-Cl-Cl CT complex, with $\mathrm{Cl}(5)-$ $\mathrm{Cl}(6)$ bond chosen as reaction coordinate (b).

\subsubsection{Bromine bound to $\mathrm{FeBr} 3$}

Bromine is a nonpolar covalent molecule, liquid under standard conditions, dark brown in color. Because of its increased reactivity to photonic excitation, bromine is stored in dark glass bottles, indicating that bromine can act as both a free radical and an oxidizing agent. In terms of its oxidizing ability, bromine is a rather weaker oxidant than $\mathrm{Cl}_{2} \mathrm{Or} \mathrm{OF}_{2}$, with a standard electrode potential of less than $1.3 \mathrm{~V}\left(E^{\circ}=1.078 \mathrm{~V}\right.$, eq. 32) [35]. In water, as a solvating agent, bromine becomes a stronger oxidizing agent with $E^{\circ}=1.098 \mathrm{~V}$ ( eq. 33) [35], with a strong indication that bromine can be polarized in the presence of protic reagents $\left(E^{\circ}=1.584 \mathrm{~V}\right.$, eq. 34) [35], making the bromonium ion a good electrophilic species. Its reactivity, either to photoexcitation or protic reagents, may come from strongly polarized p-valent orbitals, which can actively participate in the bonding process by forming either $\mathrm{CT}$ complexes or polarized adducts, with the $\mathrm{Br}-\mathrm{Br}$ bond being strongly weakened during such a process.

$$
\begin{aligned}
& \mathrm{Br}_{2(l)}+2 e^{-} \rightleftharpoons 2 \mathrm{Br}^{-} \\
& \mathrm{Br}_{2(a q)}+2 e^{-} \rightleftharpoons 2 \mathrm{Br}_{(a q)}^{-} \\
& 2 \mathrm{HBrO}+2 \mathrm{H}_{(a q)}^{+}+2 e^{-} \rightleftharpoons \mathrm{Br}_{2(l)}+2 \mathrm{H}_{2} \mathrm{O}
\end{aligned}
$$

Polarized p orbitals of bromine also react with Lewis acids in the same predicted manner, forming CT complexes that can both produce bromonium ions and interact with protic reagents, implying that acidity and electrophilicity can generally be well correlated, opening the space for future developments for these research areas in physical chemistry by raising protonic species to the level of electrophiles as well. In the following sections we will see how bromine reacts with $\mathrm{FeBr}_{3}$, as a given Lewis acid.

To our surprise, the formation of the CT complex of bromine and FeBrs occurred via a very regular bonding pattern. Iron(III) bromide started from a trigonal-planar geometry, as in all previous cases, and deformed its structure to a regular tetrahedral geometry, as predicted in many textbooks. Unusually for such a complex system, we found that all structures were identified with positive energy, which would describe a (strongly) destabilized system. On the one hand, we can agree with such an evolution of the PES profile if we consider the possibility that the bromine molecule is now polarized as well, providing an additional possibility for easy preparation of the CT complex, considering the electronic aspect. Nevertheless, the regular PES profile left two scan steps/points identified as possible CDRs, at the first and last (sixteenth) scan steps. A large energy drop was detected between the fifteenth and sixteenth scan steps $\left(\Delta E_{15-16}=38.85 \mathrm{kcal} / \mathrm{mol}\right)$, 
which is most likely responsible for the significant change in position gradients and geometry change. This actually occurred with a transition from a regular tetrahedral to a trigonal pyramidal geometry without convergence in the last scan step. To the best of our knowledge, such a transition can occur with a changed HOMO pattern with respect to the binding d orbitals. Such an energy change, even a transition to a possible more stable system, could eventually destabilize the whole CT complex, which indeed happened with respect to the preTS determination for $\mathrm{FeBr}_{3}-\mathrm{Br}_{2}$. A slight energy drop between the first and second scan steps was produced by the in-plane rotation of the $\mathrm{FeBr}_{3}$ molecule with respect to the bromine molecule, suggesting that CDR can affect such TS -like modes even by a small "irregular" gradient change, implying that the PES algorithm is quite sensitive to any kind of total energy change. Figure 19 shows how regular and CDR -free PES profiles look like.

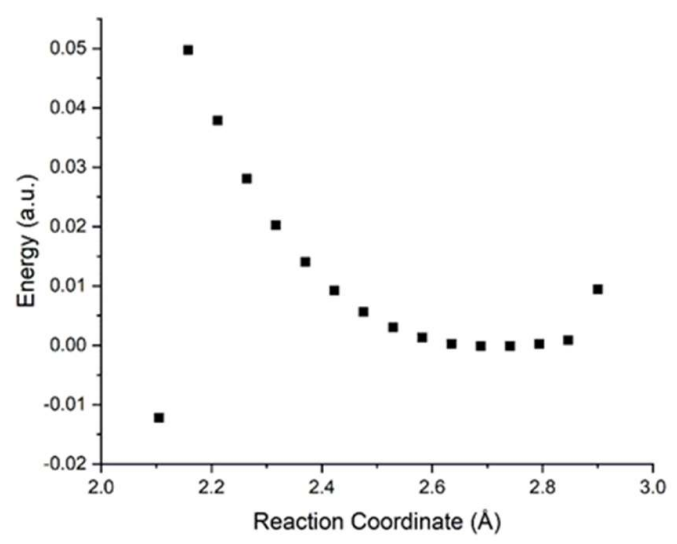

(a)

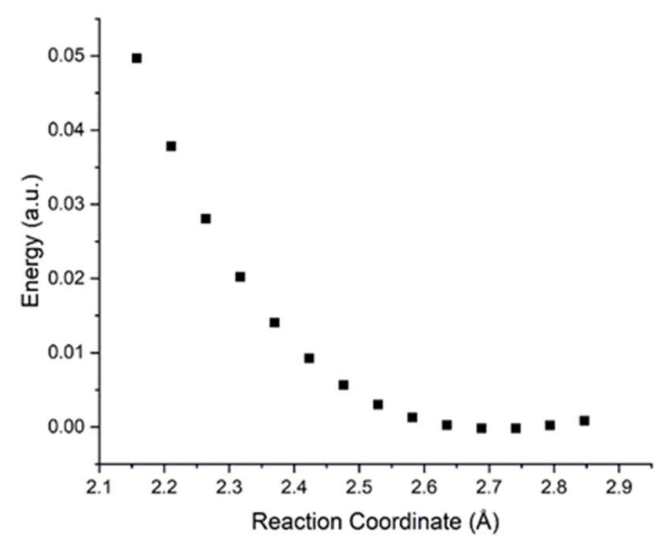

(b)

Figure 19. Regular PES profile (a) and CDR-free PES profile (b) for the bromine binding in the formation of the FeBr3$\mathrm{Br}_{2} \mathrm{CT}$ complex.

Thermochemical analysis showed that the PES profile for the FeBr3-Br2 CT complex, along the $\mathrm{Fe}-\mathrm{Br}(4)$ bond as the reaction coordinate, was characterized by the standard preTS for such complex formation, which favored its formation by lowering the free energy barrier by 25.066 $\mathrm{kcal} / \mathrm{mol}$. The total stabilization energy in such preTS characterization showed a slightly increased energy barrier, which is $23.076 \mathrm{kcal} / \mathrm{mol}$, which is an energy barrier difference small enough not to jeopardize the stability of this preTS. Such agreement with the free energy $(\Delta G)$ and stabilization energy $\left(\Delta E_{t}\right)$ may indicate that the bromine complement can be considered very stable at this stage of complex formation, but we believed that this system was further intrinsically stabilized with the surrounding bromine atoms in a thermodynamically optimal equilibrium state with respect to each other (i.e., the IR analysis agreed with the thermochemical results and revealed a single negative frequency that could not be assigned to the preTS vibration, but a weak vibration at $172.64 \mathrm{~cm}^{-1}$, which can be unambiguously characterized as a preTS frequency when the intensity and the arrangement of the displacement vectors are analyzed over the molecular map of this CT complex.

In further characterization, we again used the SOD method within the graphical method approach to predict the length of the preTS length compared to Berny's algorithm. As shown in Table 9, Berny's optimized preTS yielded a value of $2.732 \AA$, while analysis of SOD for the regular PES profile plot yielded a value of $2.258 \AA$, and with the extrapolation algorithm applied to SOD yielded a value of $2.926 \AA$. Such an occurrence in the results presented here can again provide a satisfactory level of analytical accuracy in the characterization of TS-like states, with relative errors defined as $17.35 \%$ for the regular PES profile and $7.10 \%$ for the CDR-free PES profile of this CT complex, respectively (Figure 20). 


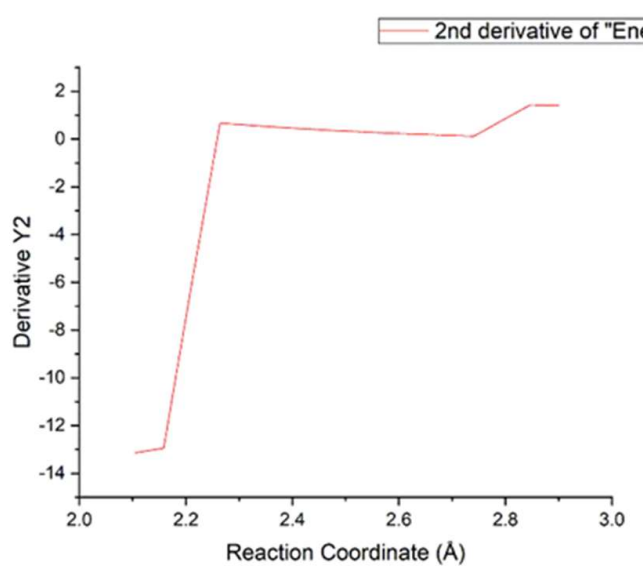

(a)

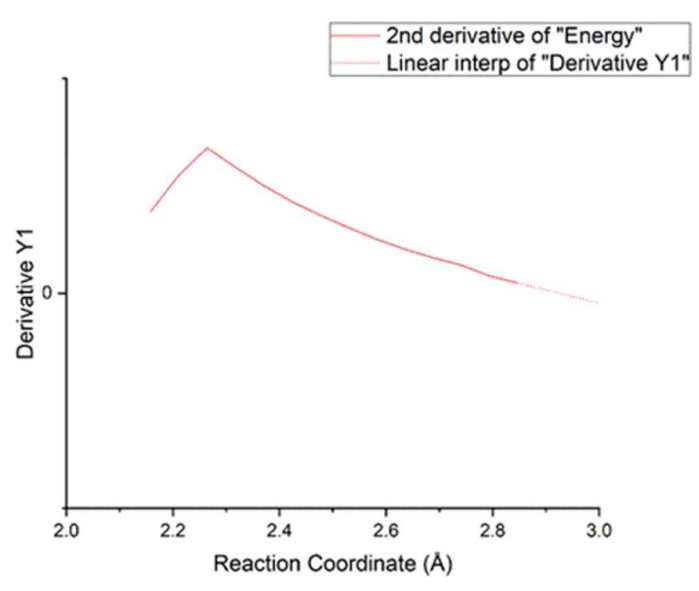

(b)

Figure 20. SOD plot for regular PES profile of $\mathrm{FeBr}_{3}-\mathrm{Br}_{2} \mathrm{CT}$ complex (a) and for CDR-free PES profile (b).

Table 9. PreTS value determined with the Berny algorithm at PM7 semiempirical theory level and the value obtained by SOD analysis for the pre-transition state in the $\mathrm{FeBr}_{3}-\mathrm{Br}_{2}$ complex.

\begin{tabular}{cccccc}
\hline & $\begin{array}{c}\text { Berny algorithm } \\
(\AA)\end{array}$ & $\begin{array}{c}\text { SOD for complete PES } \\
(\AA)\end{array}$ & $\begin{array}{c}\text { SOD for CDR-free PES } \\
(\AA)\end{array}$ & $\begin{array}{c}\text { Absolute error } \\
(\AA)\end{array}$ & Relative error (\%) \\
\hline $\begin{array}{c}\text { Fe-Br(4) } \\
\text { bond }\end{array}$ & 2.732 & 2.258 & & 0.474 (regular) & 17.35 (regular) \\
length & & & 2.926 & 0.194 (CDR- \\
free) & 7.10 (CDR-free) \\
\hline
\end{tabular}

The characterization of the bromonium ion can be characterized up to the simplest PES profile (chosen reaction coordinate was the $\operatorname{Br}(4)-\operatorname{Br}(5)$ bond), which probably shows the best fit to the theoretical prediction in PES TS characterization method, which is the best fit to the exponential flow function. The energy of the scan steps increases rapidly at first until the seventh scan step is reached. After that, a steep energy drop is observed and the system stabilizes until the end of the profile. Such a large one-time energy drop of $22.2 \mathrm{kcal} / \mathrm{mol}$ indicates changes that may have occurred in $\mathrm{FeBr}_{3}$, leading to significant position gradient changes throughout the system. Indeed, we observed an intermolecular bromine atom interaction (i.e., the previously mentioned displacement reaction) that formed another bromine molecule that moved away from the now substituted $\mathrm{FeBr}_{3}$ with respect to the positional aspect in the coordinate system. Interestingly, in this case, the geometry of the final predicted structure of the bromonium ion was observed to be similar to that found in the study of chloronium and fluoronium ions (via oxygen bonding) - formation of pentavalent iron species (i.e., tetrabromoiron(V) bromide, Figure 21). 


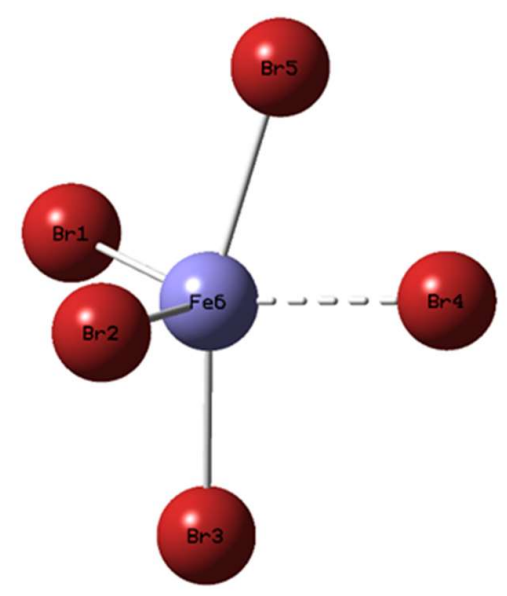

Figure 21. Tetrabromoiron(V) bromide. The dashed line indicates a bond order of less than 0.5.

Thermochemical analyzes performed with the bromonium ion TS showed exergonic behavior, as did all previous pseudoTSs. The free energy barrier for this pseudoTS was characterized as $-23.326 \mathrm{kcal} / \mathrm{mol}$, indicating that additional stabilization occurs upon formation of the bromonium ion via this Lewis acid. Moreover, it is important to note here that the total stabilization energy shows an energy barrier well below the free energy barrier, indicating that the system is stabilized rather than spontaneous, with an estimated barrier of $-33.808 \mathrm{kcal} / \mathrm{mol}$, which is in complete agreement with our predictions derived previously with F- and ClpseudoTSs that the bromonium ion was also stabilized via the ion-pair interaction under the pentavalent iron species form. On the other hand, something curious ran through the characterization of these pseudoTSs - the inconsistency between the pseudoTS results and the PES profile of bromonium ion formation. Both were characterized at the PM7 semiempirical level of theory, but the displacement reaction showed up in the analysis of PES, while the pentavalent iron species was detected in the pseudoTS. Therefore, we suggest that the pentavalent iron species identified here as pseudoTS is a much more time-dependent species than in the case of chloronium or fluoronium ions. Moreover, we have obtained sufficient evidence that this is a unique case in which rapid exchange between bromine atoms occurs continuously, leaving no stable species that can be said with certainty to be responsible for the formation of halonium (i.e., bromonium) ions. This concluded our analyzes at this point. The analysis of IR showed the same result as in the previous cases, isolating a single negative frequency not corresponding to the pseudoTS at $81.26 \mathrm{~cm}^{-1}$, with possible positive frequencies corresponding to such a pseudoTS at several values ranging from +109.11 to $+424.81 \mathrm{~cm}^{-1}$. Analysis of SOD yielded a value of $2.827 \AA$, which is in solid agreement with the Berny approximation of $2.780 \AA$. No CDR was identified, resulting in a relative error in the pseudoTS determination for the bromonium ion of $1.69 \%$, which is safely within the exact analytical interval $(\leq 2 \%)$, within the given confidence level of $99 \%(p=0.01)$. Table 10 summarizes all the details of the pseudoTS for the bromonium ion.

Table 10. PseudoTS value determined by Berny algorithm at PM7 semiempirical level of theory and that obtained by SOD analysis for the pre-transition state in $\mathrm{FeBr} 3 \mathrm{Br}-\mathrm{Br}$ complex.

\begin{tabular}{lccccc}
\hline & $\begin{array}{c}\text { Berny algorithm } \\
(\AA)\end{array}$ & $\begin{array}{c}\text { SOD for complete PES } \\
(\AA)\end{array}$ & $\begin{array}{c}\text { SOD for CDR-free PES } \\
(\AA)\end{array}$ & $\begin{array}{c}\text { Absolute error } \\
(\AA)\end{array}$ & Relative error $(\%)$ \\
\hline $\begin{array}{c}\operatorname{Br}(4)-\operatorname{Br}(5) \\
\text { bond } \\
\text { length }\end{array}$ & 2.780 & 2.827 & ND $^{*}$ & 0.047 (regular) & 1.69 (regular) \\
\hline
\end{tabular}

${ }^{*} \mathrm{ND}=$ Not Defined 


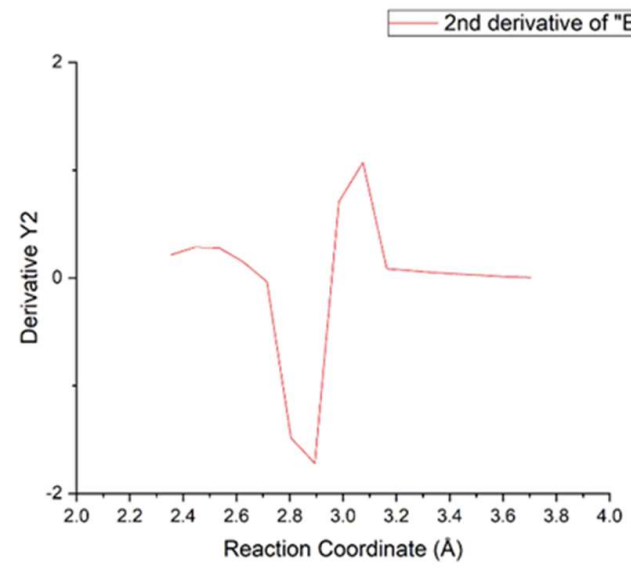

(a)

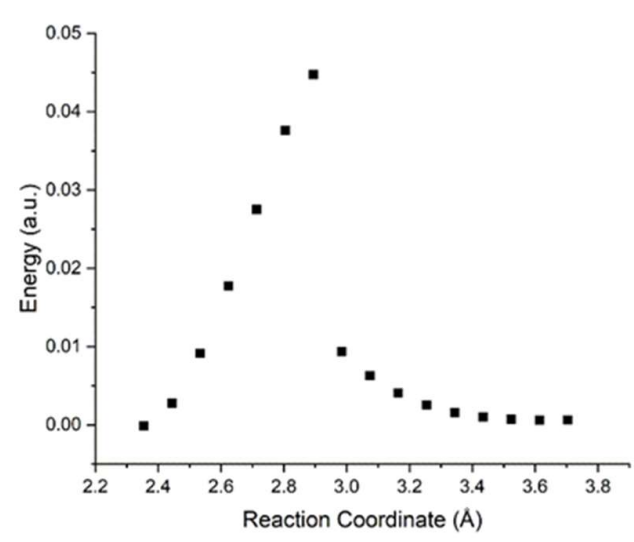

(b)

Figure 22. SOD plot for $\mathrm{FeBr}_{3} \mathrm{Br}-\mathrm{Br} \mathrm{CT}$ complex (a) and regular PES profile for $\mathrm{FeBr} 3 \mathrm{Br}-\mathrm{Br} \mathrm{CT}$ complex, with $\mathrm{Br}(4)-\mathrm{Br}(5)$ bond chosen as reaction coordinate $(b)$.

\subsubsection{Iodine(I)-chloride bound to $\mathrm{FeBr} 3$}

Iodine(I) chloride $(\mathrm{ICl})$ is a reddish-brown liquid with high density $(\mathrm{Q}=3.10 \mathrm{~g} / \mathrm{mL})[44]$, which has an experimental bond length of $2.32 \AA$. ICl contains an iodine atom in the +1 oxidation state and a chlorine atom in the -1 oxidation state, which can subsequently react in many electrophilic quenching reactions, with iodine characterized as highly electrophilic. However, the electronic picture of $\mathrm{ICl}$ is somewhat more complex, with the valent p-orbitals of iodine and chlorine shifted at different quantum levels, which greatly alters their nucleophilic (HOMO) character in different reactions, depending on the solvent in which the reaction takes place. The proposed reactivity pathway was that in strongly aprotic or apolar solvents - iodine is most likely to react, while in protic or less aprotic solvents - chlorine is most likely to be nucleophilic. Within this statement there is a solid boundary line for HOMO activity, and it is interchangeable, depending on the solvent chosen experimentally, but also on the intensity and nature of the species interacting with ICl! In toluene, the electron density picture (MEP, Figure 23a) made at the M06L/6-311+G(3d2f)/SDD level of theory shows a classical predicted model for the electron density disposition - a larger electron density over chlorine and a much smaller one over iodine, especially at the axial position (together with the I-Cl bond). The predicted HOMOLUMO gap of $-2.737 \mathrm{eV}$ is also consistent with the character of a strongly covalent compound and is electrophilic/nucleophilic rather than basic (on chlorine). Such behavior is likely to be further favored by interaction with an apolar solvent such as toluene $(\varepsilon=2.374)$ [45]. Figures $23 \mathrm{~b}$ and $23 \mathrm{c}$ show HOMO and LUMO of ICl, respectively. In the electric field, $\mathrm{ICl}$ can act as a strong oxidant and reduce to iodine, with $E^{\circ}=1.220 \mathrm{~V}$ [35].

With respect to the $\mathrm{FeBr}_{3}-\mathrm{ClI}$ complexation, we performed a PES profile job to see whether or not the interactions were as previously described, taking into account the toluene interactions. The Fe-Cl bond was chosen as the reaction coordinate, as it is believed to be formed. The PES profile, performed with the semiempirical PM7 functional, showed a fairly smooth profile with a single decreasing energy point. Along the reaction coordinate, the bond between iron and chlorine became shorter and the energy gradients worked well, considering that double reactivity is generally observed in $\mathrm{ICl}$. In fact, the chlorine atom, although having a lower HOMO than the iodine atom, used the reactivity pathway in this case, leading to the tribromochloroferrate(III) complex. We believe that this result in reactivity came about due to the overall electronic effect (encompassing both HOMO and LUMO together) that easily distinguishes chlorine as a nucleophilic species. 


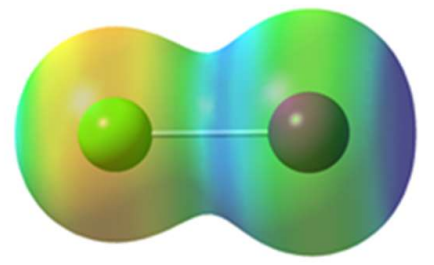

(a)

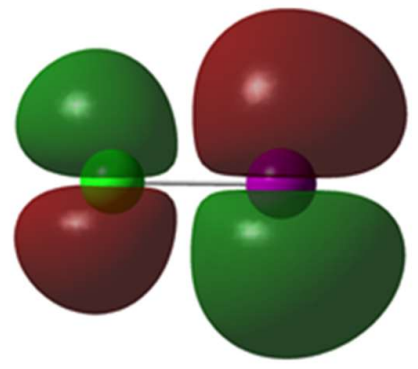

(b)

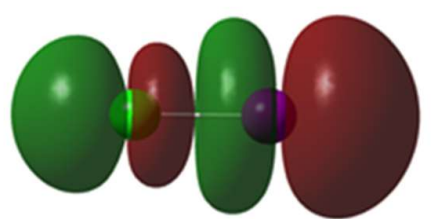

(c)

Figure 23. ICl molecule mapped as a function of electron density (MEP legend: red - most electron dense; blue - least electron dense) (a), HOMO eigenvector (b) and LUMO eigenvector (c).

Between the sixth and seventh scan steps, there was a decrease in the energy of the system, indicating that a preTS can be found for this complex (Figure 24). The energy gap value of 26.882 $\mathrm{kcal} / \mathrm{mol}$ suggests a larger transformation along the given reaction coordinate, and that significant positional gradients are likely altered. This conjecture has proven true, with the transition from tetrahedral symmetry to trigonal pyramid identified. This geometry change has been observed before, but there is no direct evidence to say with clarity why this geometry change occurred. Iron(III) is able to model its orbitals, in particular HOMO, while the LUMO orbital on iron(III) is larger than $d_{z^{2}}$, most likely due to the fact that the iron(III) species can have both a high spin iron center and a low spin one. Since it is a high spin center, as predicted for monomeric $\mathrm{FeBr}$, we concluded that spin differentiation also affects this sudden transition from tetrahedral to trigonal pyramid. Anyway, the presence of a single inflection point where the energy decrease is straight, we can say that this transition in energy can be characterized as a kind of CDR phenomenon that we would call pseudoCDR. As we have observed, pseudoCDRs are treated normally and can bypass the detection of the TS point as efficiently as regular CDRs.

Therodynamic analyzes revealed no negative frequencies, only a relatively weak frequency at $514.69 \mathrm{~cm}^{-1}$ characterizing the $\mathrm{Fe}-\mathrm{Cl}$ stretching band in a trigonal pyramidal geometry. The exergonic behavior of such preTS would be attributed to an energy barrier for such preTS formation of $-23.498 \mathrm{kcal} / \mathrm{mol}$, suggesting that chlorine bonding is rather favored and a possible ferrate ion could be a pool for potential iodonium ion formation. The total stabilization energy settled at $-49.457 \mathrm{kcal} / \mathrm{mol}$, which is well above chlorine or bromine CT complex formation (cf. preTS for chlorine and bromine systems). With respect to this last electrophiloid studied, we must say that we did not look at the transition state pathway to characterize its spontaneity, but at the energies for TSs themselves! This hint would assure us that an accurate analysis of the intrinsic stabilization tendencies or spontaneity in the formation and existence of such complexes would suffice in all research of this kind. SOD analyzes were performed, but since no significant CDR was found, we concluded that the preTS value of the reaction coordinate was $2.011 \AA$. In contrast, Berny's algorithm suggested that this distance in preTS is reached at $2.121 \AA$, which is the result whose outcome, measured as relative error in prediction, was calculated to be $5.19 \%$, again considered to be within the highest confidence interval $(\mathrm{p}=0.01)$ within the statistics of the analytical analyzes. Table 11 summarizes the data on this complex. 
Table 11. PreTS value determined with the Berny algorithm at the PM7 semiempirical theory level and the value obtained by SOD analysis for the pre-transition state in the $\mathrm{FeBr}_{3}-\mathrm{ClI}$ complex.

\begin{tabular}{|c|c|c|c|c|c|}
\hline & $\begin{array}{c}\text { Berny algorithm } \\
(\AA)\end{array}$ & $\begin{array}{l}\text { SOD for complete PES } \\
\qquad(\AA)\end{array}$ & $\begin{array}{l}\text { SOD for CDR-free PES } \\
\qquad(\AA)\end{array}$ & $\begin{array}{c}\text { Absolute error } \\
(\AA)\end{array}$ & Relative error (\%) \\
\hline $\mathrm{Fe}-\mathrm{Cl}$ & \multirow{3}{*}{2.121} & \multirow{3}{*}{2.011} & \multirow{3}{*}{$\mathrm{ND}^{*}$} & \multirow{3}{*}{0.110 (regular) } & \multirow{3}{*}{5.19 (regular) } \\
\hline bond & & & & & \\
\hline length & & & & & \\
\hline
\end{tabular}

${ }^{*} \mathrm{ND}=$ Not Defined.

It may be interesting to note what happens in the formation of iodonium ions after preTS and appropriate Fe-Cl CT complexation (Figures 25 and 26). Here we have performed the PES job at the PM7 semiempirical theory level to obtain the results within this methodology. Nevertheless, the PES profile follows the same pattern as in the previous cases, although it is useful to point out the slight decrease in energy at the second scan step, which at first sight could lead to the assumption that the first scan step acts as a potential CDR. Further analysis showed a regular change in the energy gradient, with an increase in the total energy of the profile. At the tenth scan step, a sudden steep drop in energy occurred. Analysing the PES profile of iodonium ion formation, we found convergence only in IBr molecule formation and $\mathrm{FeBr}_{2} \mathrm{Cl}$ regeneration. On the other hand, $\mathrm{FeBr}_{3}$ reconstitutes after catalysis of the EAS reaction, and this is the point we have relied on in this part of the discussion.

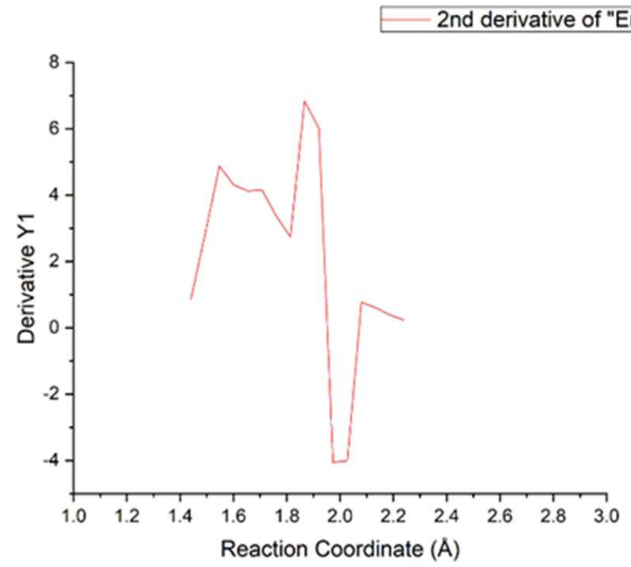

(a)

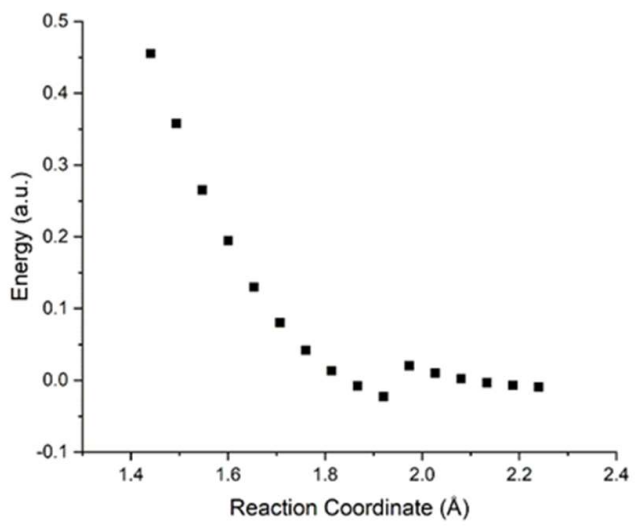

(b)

Figure 24. SOD plot for FeBr3-CII CT complex (a) and regular PES profile for $\mathrm{FeBr}_{3}-\mathrm{ClI} \mathrm{CT}$ complex, with Fe-Cl bond chosen as reaction coordinate $(\mathrm{b})$.

We believe that the IBr molecule, although less polarized, can serve as an iodonium ion pool, allowing the bromide ion to act as a better leaving group in the toluene and the possibility of $\mathrm{FeBr}_{3}$ regeneration as well as chloride ion expulsion throughout the reaction process. Beginning with the eleventh scan step, we noticed something that may be important to this discussion and that is consistent with our previous assumption. Namely, starting at the eleventh scan step, we noticed that the I-Br bond is more polarized, with positive energy gradients observed. This fact probably causes the I-Br bond to break as a function of time, releasing bromide ions and returning the catalytic system to the way it was at the beginning. 


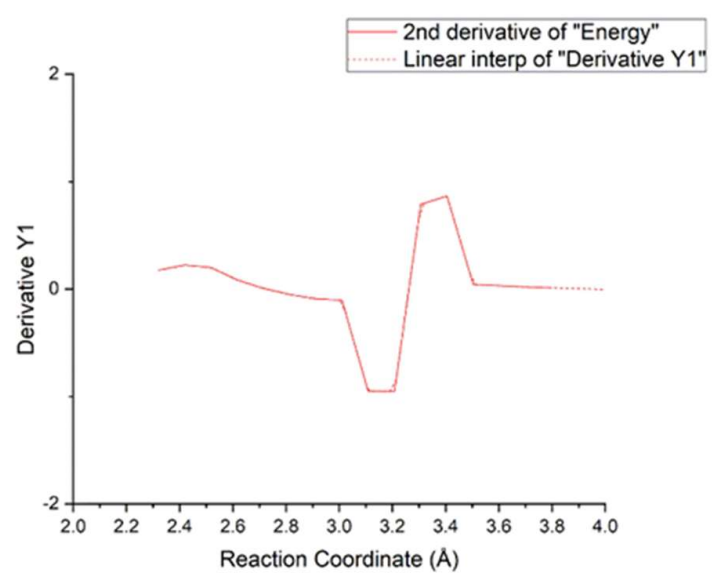

(a)

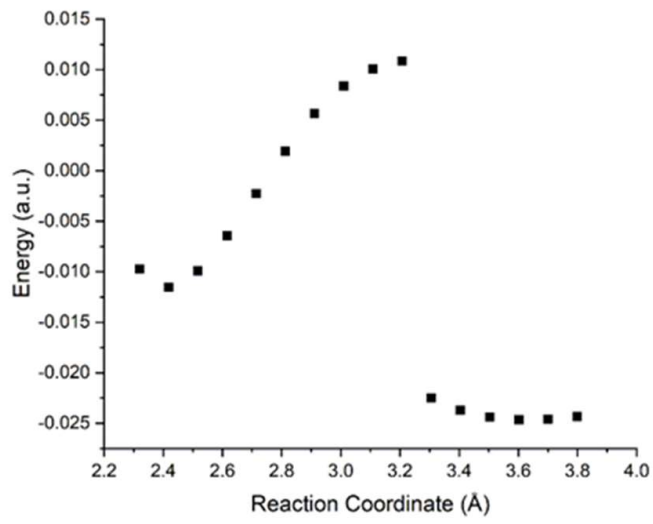

(b)

Figure 25. SOD plot for $\mathrm{FeBr}_{3} \mathrm{Cl}-\mathrm{I} \mathrm{CT}$ complex (a) and regular PES profile for $\mathrm{FeBr}_{3} \mathrm{Cl}-\mathrm{I} \mathrm{CT}$ complex, with Cl-I bond selected as reaction coordinate $(b)$.

Thermodynamic analysis of iodonium ion formation revealed several pieces of information. First, a single negative frequency at $4.75 \mathrm{~cm}^{-1}$ was identified, which we normally neglect and attribute to noise in the computational algorithm. Further analysis gave a better insight into the positioning of the bands of the IR spectrum and defined that the I-Cl bond is rather not seen in the IR spectrum, not even in the region of the positive frequencies. However, analyzing the displacement vectors, we were able to find a frequency most similar to the I-Cl stretching band, appearing at $114.97 \mathrm{~cm}^{-1}$, but with very weak intensity. On the other hand, again to our surprise, we found a rather strong stretching band of the Fe-Br bond (Figure 27) at $188.94 \mathrm{~cm}-1$. Moreover, as a side confirmation of $\mathrm{Fe}-\mathrm{Cl}$ non-covalent bond, we found an oscillation with very strong intensity at $434.21 \mathrm{~cm}^{-1}$, indicating the possibility of formation of iodonium ions with $\mathrm{ICl}$ as electrophiloid.

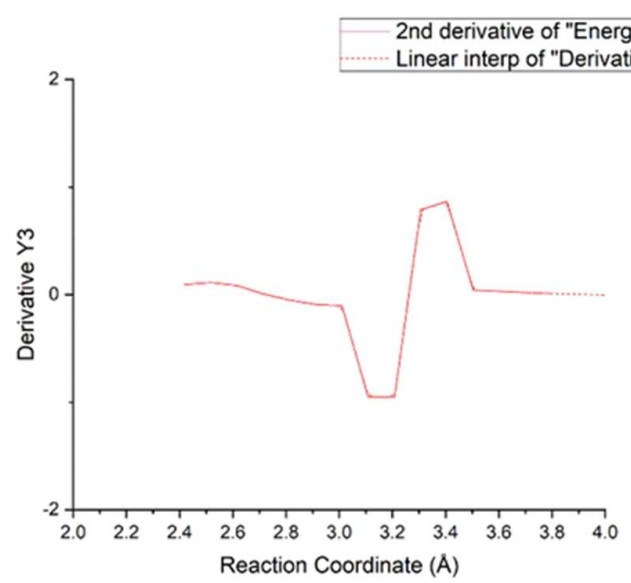

(a)

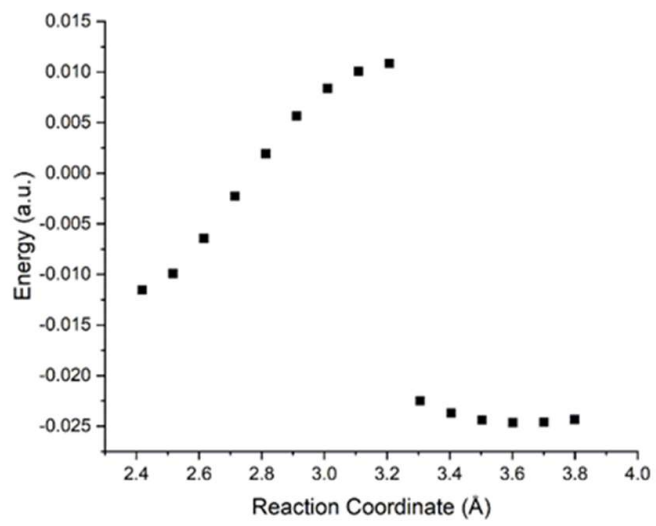

(b)

Figure 26. CDR-free SOD plot for $\mathrm{FeBr}_{3} \mathrm{Cl}-\mathrm{I} \mathrm{CT}$ complex (a) and CDR-free PES profile for FeBr3Cl-I CT complex, with $\mathrm{Cl}-\mathrm{I}$ bond chosen as reaction coordinate $(\mathrm{b})$.

Further thermochemical analyses concerned the overall stability and spontaneity of this pseudoTS formation. The spontaneous formation of such CT complex in pseudoTS occurred with a lower energy barrier, defined here as $-23.803 \mathrm{kcal} / \mathrm{mol}$, which is in the range where other discussed complexes are found. Although a significant decrease in the energy barrier can be 
considered when speaking of a general energy stabilisation, which sets its value at -38.803 $\mathrm{kcal} / \mathrm{mol}$, which is among the highest for the complexes studied.

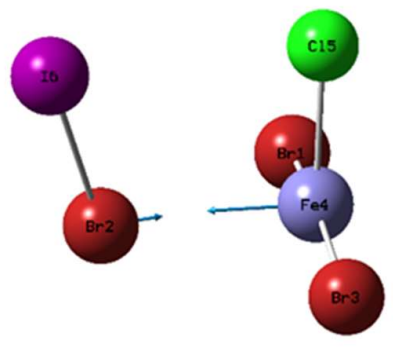

Figure 27. Displacement vectors (blue arrows) for Fe-Br stretching vibration.

SOD analysis of the regular and CDR-free profiles yielded results that differed by $\approx 1 \AA$. This difference drastically increased the relative error of the pseudoTS determination. By applying the extrapolation algorithm to the given SODs, we obtained much better results, within an error of $0.3 \AA$, which was even smaller for CDR-corrected profiles. Table 12 contains summarized data on all important bond lengths and relative errors in the given approximations. Already the values of 7.72 and $7.89 \%$ as relative error prove the good applicability of the SOD method within the graphical method even for sensitive systems with multiple transformations during energy scanning.

Table 12. PseudoTS value determined with the Berny algorithm at the PM7 semiempirical level of theory and that obtained by the SOD analysis for the pseudo-transition state in the FeBr3Cl-I complex.

\begin{tabular}{|c|c|c|c|c|c|}
\hline & $\begin{array}{c}\text { Berny algorithm } \\
(\AA)\end{array}$ & $\begin{array}{l}\text { SOD for complete PES } \\
\qquad(\AA)\end{array}$ & $\begin{array}{l}\text { SOD for CDR-free PES } \\
(\AA ̊)\end{array}$ & $\begin{array}{l}\text { Absolute error } \\
\qquad(\AA)\end{array}$ & Relative error (\%) \\
\hline $\mathrm{Cl}-\mathrm{I}$ & & & & 0.283 (regular) & \\
\hline $\begin{array}{l}\text { bond } \\
\text { length }\end{array}$ & 3.587 & 3.304 & 3.310 & $\begin{array}{c}0.277 \text { (CDR- } \\
\text { free) }\end{array}$ & 7.72 (CDR-free) \\
\hline
\end{tabular}

\section{Experimental}

All structures were analyzed and optimized with Gaussian16 program code [46]. All structures were created and maintained using the GaussView 6 graphic user interace program [47]. The initial structures for each electrophiloid discussed in this manuscript were molecularly pre-optimized using Universal Force Field (UFF) to obtain a better initial structure for further optimization. A symmetry algorithm was then used to assign each structure to the correct symmetry group. In some cases, the symmetry keyword was intentionally turned off to avoid entropy errors that could have resulted from inappropriate use of this keyword. In all other cases, especially when optimizing electrophiloids $\left(\mathrm{OF}_{2}, \mathrm{Cl}_{2}\right.$ and $\left.\mathrm{ICl}\right)$, the correct symmetry group was regularly assigned and used in the respective input file(s).

For all structures, the solvent keyword was used to estimate given solvent interactions to optimize the structures/complexes/states. In accordance with the name of the topic, we chose toluene as the solvent, due to its slightly increased reactivity in electrophilic aromatic substitution reactions, being a representative aromatic compound (i.e. very common in the experimental sense), so that it could serve as a relevant reference point for the interactions studied, as well as its very low dielectric constant, which was chosen intentionally to see how the analyzed complexes would behave in such a medium. We did not succeed in optimizing the $\mathrm{FeBr}_{3}$ monomer in the toluene medium at the DFT (M06L) theory level, even at a higher maximum cycle keyword, so we optimized the same 
at the same theory level but in the gas phase. Considering the purpose of the monomer calculations performed, we thought that it would be satisfactory to perform such a task in the gas phase.

The choice of the semiempirical level of theory was, as mentioned in the previous text, the trade-off for the ratio of accuracy and computational cost. PM7 [48], as the currently most accurate semiempirical functional, was the best candidate for the calculations, for the results presented here. Of course, the potential energy scan, together with the intrinsic reaction coordinate (IRC) and molecular dynamics (MD) tasks, is one of the most complicated tasks that can be performed on today's professional computational programs, so the choice of PM7 was all the more encouraging also in view of this fact. In addition, the meta-GGA functional M06L was chosen for all iron-based compounds, as it is better implemented than other Truhlar functions [38]. M06L was also used for aluminum compounds and complexes for comparison, although its performance for main group metals was worse than that of other, e.g., hybrid meta-GGAs, such as M062X or M06HF. Nevertheless, the obtained results showed a good agreement, despite all the previous remarks. For $\mathrm{OF}_{2}$, a very high accuracy of the obtained data should be achieved, mainly because of the orbital shift, which was the goal in this study within the given objective. For all types of transition states, the semiempirical PM7 functional was used, with the implementation of the extra-quadratic convergence algorithm for SCF frequency calculations, due to the complexity of the analyzed systems and the partial geometries of non-covalent nature (it has the tendency toward hardly converging issues).

The choice of basis set ( for DFT functional level optimized structures) was determined due to the need to achieve greater accuracy. Therefore, the fivefold zetavalence basis set (cc-pV5Z) was implemented on OF2. The good correlation with few additional sets of polarized functions gave a better insight into the electronic properties, as well as possible orbital interactions intra- or intermolecularly. The choice of $6-311+G(d)$ and $6-311+G(3 \mathrm{~d} 2 \mathrm{f})$ Pople triple zeta basis sets for iron $(\mathrm{V})$ species and chlorine/chloride/chloronium ions, respectively, should provide a good methodological link in this type of studies.

The B3LYP hybrid GGA functional was used for the thermodynamic analyses of iron $(\mathrm{V})$ (which was not ultimately intended to be part of this manuscript), as it has generally been shown to work well for all of the first row transition state metals and the rest of the main group elements, and higher accuracy was required for TS identification in these compounds. No additional IRC analyses were performed for these systems, as they were well analysed by displacement vector analyses on given TSs and showed interesting results. In the Supplementary Materials only the Cartesian coordinates of the studied structures were given. The results will be published in a later manuscript.

SOD analyses were performed by intersecting the zero value of the $y$-axis with the function of the 2 nd derivative of energy (heat of formation). In cases where such a curve line is (more complex), intersecting the function at the zero value may result in obtaining multiple values. The mean equation $\left(x_{m}\right.$, eq. 35$)$ resolves the best-fit prediction point for such a transition state, which is generally independent of the number of detected CDR (s):

$$
x_{m}=\frac{\sum_{i=1}^{k} x_{i}}{k}
$$

\section{Conclusions}

PES analysis are promising algorithms in theoretical and computational chemistry, the analysis of which is very complex, which can sometimes complicate the work of physical organic/theoretical chemists. In modern chemical science, there are no essays on the detection of theoretically given charge transfer states. Although it is difficult to analyse, we manage to solve the preliminary stage of such analysis by developing and implementing the approach of graphical methodology in this kind of scan. The graphical methodology includes the FOD and SOD methods, which can accurately account for microscopic processes that occur as a function of time, as well as microscopic processes 
that are based on reversibility and involve the movement of electrons between orbitals within the FMO region or within intrinsic shells.

Using semiempirical PM7 methods, we were able to discover a type of noncovalent interaction that appears to control the outcome of the experimental procedure in these systems. In the studies carried out with PM7 functionality, we have succeeded in finding and localizing interactions that were not known in the chemical community since the recently published work on the topic of the bimetallic catalytic process between the interactions between transition and main group metals [42,49]. Based on the same mechanistic principles, we have solved for the first time a stabilizing interaction between transition metal - halogen ion pair where the halogen serves as the electrophilic species, which is different from the textbook predictions at the theoretical level for this type of complexes/ catalytic species. This has encouraged us to make a further contribution to the chemistry of transition metal catalysis.

In conclusion, we think that the current topics in this research area as well as this manuscript will "open the door" for new perspectives in this field, but also help in the development of new methods and methodologies in the recent future for even finer system analysis, in catalysis or other areas of chemistry.

Supplementary Materials: The following are available online at www.mdpi.com/xxx/s1, Cartesian coordinates of the optimized structures studied and additional coordinates to structures and transition states on iron $(\mathrm{V})$ species.

Author Contributions: Conceptualization, N.S. and J.C.; methodology, N.S..; validation, N.S. and J.C.; formal analysis, N.S.; investigation, N.S. and J.C.; resources, J.C.; data curation, N.S.; writingoriginal draft preparation, N.S. and J.C.; project administration, J.C.; funding acquisition, J.C. All authors have read and agreed to the published version of the manuscript.

Funding: This research was funded by Slovenian Research Agency (Research Core Funding Grant P1-0230).

Acknowledgments: We thank Chemical Institute of Slovenia (Dr. Jernej Stare) for permission to perform computations on HPC supercomputer clusters.

Conflicts of Interest: The authors declare no conflict of interest.

\section{References}

1. Crampton, M.R. Aromatic Substitution. In: Organic Reaction Mechanisms 2016; Knipe, A.C., Ed.; Wiley \& Sons, 2020, pp. $247-335$.

2. Anslyn, E.V.; Dougherty, D.A. Modern Physical Organic Chemistry. Mill Valley, California: University Science Books, 2006, p. 1099.

3. $\quad$ Bruckner, R. Advanced Organic Chemistry; Elsevier, 2002, p. 636.

4. Hubig, S.M.; Kochi, J.K. Structure and Dynamics of Reactive Intermediates in Reaction Mechanisms. $\sigma$ - and $\pi$-Complexes in Electrophilic Aromatic Substitutions. J. Org. Chem., 2000, 65, 6807-6818.

5. Du, Z.; Shao, Z. Combining transition metal catalysis and organocatalysis - an update. Chem. Soc. Rev. 2013, 42, 1337-1378. Doi: 10.1039/C2CS35258C.

6. Galabov, B.; Nalbantova, D.; Schleyer, P.v.R.; Schaefer III, H.F. Electrophilic Aromatic Substitution: New Insights into an Old Class of Reactions. Acc. Chem. Res. 2016, 49, 1191-1199. Doi: 10.1021/acs.accounts.6b00120.

7. Galabov, B.; Koleva, G.; Simova, S.; Hadijeva, B.; Schaefer III, H.F. Arenium ions are not obligatory intermediates in electrophilic aromatic substitution. Proc. Natl. Acad. Sci. U. S. A., 2014, 111, 10067-10072. Doi: 10.1073/pnas.1405065111.

8. Rouvray, D.H.; King, R.B. Topology in Chemistry: Discrete Mathematics of Molecules; Horwood Publishing: West Sussex, England, 2002, p. 386.

9. Fleming, I. Molecular Orbitals and Organic Chemical Reactions; Wiley \& Sons: West Sussex, 2010, p. 529.

10. Cramer, C.J. Essentials of Computational Chemistry: Theories and Models, $2^{\text {nd }}$ ed.; Wiley, 2004, p. 618.

11. Corey, E.J.; Chelg, X.-M. The Logic of Chemical Synthesis; Wiley \& Sons: New York, 1995, p. 464.

12. Tawfik, A.N.; Diab, A.M. A review of the generalized uncertainty principle. Rep. Prog. Phys. 2015, 78, 126001. Doi: 10.1088/00344885/78/12/126001.

13. Ostlund, S.; Pandit, R.; Rand, D.; Schellnhuber, H.J.; Siggia, E.D. One-Dimensional Schrödinger Equation with an Almost Periodic Potential. Phys. Rev. Lett. 1983, 50, 1873. Doi: 10.1103/PhysRevLett.50.1873.

14. Amyes, T.L.; Richard, J.P. Specificity in Transition State Binding: The Pauling Model Revisited. Biochemistry 2013, 52, $2021-2035$. Doi: 10.1021/bi301491r. 
15. Henkelman, G.; Jónsson, H. A climbing image nudged elastic band method for finding saddle points and minimum energy paths. J. Chem. Phys. 2000, 113, 9901-9904. Doi: 10.1063/1.1329672.

16. Govind, M.; Petersen, M.; Fitzgerald, G.; King-Smith, D.; Andzelm, J. A Generalized Synchronous Transit Method for Transition State Location. Comput. Mater. Sci. 2003, 28, 250-258. Doi: 10.1016/S0927-0256(03)00111-3.

17. Becke, A. D. A new mixing of Hartree-Fock and local density-functional theories. J. Chem. Phys. 1993, 98, 1372-1377.

18. Rozanska, X. et al. High-Throughput Calculations of Molecular Properties in the MedeA Environment: Accuracy of PM7 in Predicting Vibrational Frequencies, Ideal Gas Entropies, Heat Capacities, and Gibbs Free Energies of Organic Molecules. J. Chem. Eng. Data 2014, 59, 3136-3143. Doi: 10.1021/je500201y.

19. All fluorine agents are electrophiloid fluorine agents, like nitrosyl-fluoride, nitryl-fluoride, IF5 etc.

20. Law of electron density conservation is analogous Law to that of energy conservation. Therefore, one can draw the common line between these two.

21. Sen, A.; Srivastava M. Regression Analysis: Theory, Methods, and Applications; Springer-Verlag: NY, USA, 1990, p. 347.

22. Abdi, H. Partial least squares regression and projection on latent structure regression (PLS Regression). WIREs Comput. Stat. 2010, 2, 97-106. Doi: 10.1002/wics.51.

23. Menke, W. Review of the Generalized Least Squares Method. Surv. Geophys. 2015, 36, 1-25. Doi: 10.1007/s10712-014-9303-1.

24. Guevara-Garcia, A.; Ayers, P.W. et al. Pointing the way to the products? Comparison of the stress tensor and the second-derivative tensor of the electron density. J. Chem. Phys. 2011, 134, 234106. Doi: 10.1063/1.3599935.

25. Fike, J.A.; Alonso, J.J. The Development of Hyper-Dual Numbers for Exact Second-Derivative Calculations. 49th AIAA Aerospace Sciences Meeting including the New Horizons Forum and Aerospace Exposition; Orlando, Florida, USA, January $44^{\text {th }}-$ January $7^{\text {th }}$ 2011. Doi: 10.2514/6.2011-886.

26. Lu, T.; Chen, F. Bond Order Analysis Based on the Laplacian of Electron Density in Fuzzy Overlap Space. J. Phys. Chem. A 2013, 117, 3100-3108. Doi: 10.1021/jp4010345.

27. Śmiga, S.; Fabiano, E.; Constantin, L.A.; Della Sala, F. Laplacian-dependent models of the kinetic energy density: Applications in subsystem density functional theory with meta-generalized gradient approximation functionals. J. Chem. Phys. 2017, 146, 064105. Doi: 10.1063/1.4975092.

28. Togneti, V.; Joubert, L. Electron density Laplacian and halogen bonds. Theor. Chem. Acc. 2015, 134, 90. Doi: 10.1007/s00214-0151685-8.

29. Duarte, D.J.R.; Sosa, G.L.; Peruchena, N.M. Nature of halogen bonding. A study based on the topological analysis of the Laplacian of the electron charge density and an energy decomposition analysis. J. Mol. Model. 2013, 19, 2035-2041. Doi: 10.1007/s00894-012-1624-8.

30. McNamara, J.P.; Sundararajan, M.; Hillier, I.H.; Ge, J.; Campbell, A.; Morgado, C. Can the Semiempirical PM3 Scheme Describe Iron-Containing Bioinorganic Molecules? J. Comput. Chem. 2006, 27, 1307-1323. Doi: 10.1002/jcc.20403.

31. Christian, J.D.; Gregory, N.W. Vapor-phase absorption characteristics of iron(III)bromide and iron(III)chloride in the ultraviolet-visible region. J. Phys. Chem. 1967, 71, 1579-1583. Doi: 10.1021/j100865a005.

32. Akdeniz, Z.; Pastore, G.; Tosi, M.P. Structure and binding of molecular clusters of trivalent metal halides in an ionic model. Int. Centre Theor. Phys. 1997, 177.

33. Tomifuji, R.; Kurahashi, T.; Matsubara, S. Asymmetric Aza-Diels-Alder Reaction with Ion-Paired-Iron Lewis AcidBrønsted Acid Catalyst. Chem. Eur. J. 2019, 25, 8987-8991. Doi: 10.1002/chem.201901563.

34. Hanelt, S.; Friedrich, J.F.; Orts-Gil, G.; Meyer-Plath, A. Study of Lewis acid catalyzed chemical bromination and bromoalkylation of multi-walled carbon nanotubes. Carbon 2012, 50, 1373-1385. Doi: 10.1016/j.carbon.2011.11.009.

35. Harris, D.C. Quantitative Chemical Analysis, $7^{\text {th }}$ ed.; Freeman \& Co.: NY, USA, 2007, p. 828.

36. Cotton, F.A.; Wilkinson, G. Advanced Inorganic Chemistry, $3^{\text {rd }}$ ed.; Wiley \& Sons, 1972, p. 1171.

37. Glendening, E.D.; Reed, A.E.; Carpenter, J.E.; Weinhold, F. NBO Version 3.1.

38. Wang, Y. et al. Revised M06 density functional for main-group and transition-metal chemistry. Proc. Natl. Acad. Sci. USA 2018, 115, 10257-10262. Doi: 10.1073/pnas.1705670114.

39. Møller, C.; Plesset, M.S. Note on an Approximation Treatment for Many-Electron Systems. Phys. Rev. 1934, 46, 618-622. Doi: $10.1103 \% 2$ FPhysRev.46.618.

40. Suleimanov, Y.V.; Green, W.H. Automated Discovery of Elementary Chemical Reaction Steps Using Freezing String and Berny Optimization Methods. J. Chem. Theory Comput. 2015, 11, 4248-4259. Doi: 10.1021/acs.jctc.5b00407.

41. Hargittai, M.; Kolonits, M.; Tremmel, J.; Fourquet. J.; Ferey, G. The molecular geometry of iron trifluoride from electron diffraction and a reinvestigation of aluminum trifluoride. Struc. Chem. 1990, 1, 75-78. Doi: 10.1007\%2FBF00675786.

42. Similar bimetallic catalytic system has been characterized in pioneering stage recently: Escomel, L.; Camp, C. et al. Strongly

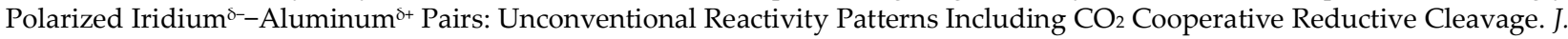
Am. Chem. Soc. 2021, 143, 4844-4856. Doi: 10.1021/jacs.1c01725.

43. Flick, J.C. et al. Accurate Prediction of Noncovalent Interaction Energies with the Effective Fragment Potential Method: Comparison of Energy Components to Symmetry-Adapted Perturbation Theory for the S22 Test Set. J. Chem. Theory Comput. 2012, 8, 2835-2843. Doi: 10.1021/ct200673a. (+ correction paper)

44. Brisbois, R. G.; Wanke, R. A.; Stubbs, K. A.; Stick, R. V. Iodine Monochloride. In: Encyclopedia of Reagents for Organic Synthesis; Wiley \& Sons, 2004. Doi: $10.1002 \% 2 F 047084289$ X.ri014.

45. Gaussian, Inc. https://gaussian.com/scrf/. (accessed April 12 ${ }^{\text {th }}$ 2021). 
46. Gaussian 16, Revision B.01, Frisch, M. J.; Trucks, G. W.; Schlegel, H. B.; Scuseria, G. E.; Robb, M. A.; Cheeseman, J. R.; Scalmani, G.; Barone, V.; Petersson, G. A.; Nakatsuji, H.; Li, X.; Caricato, M.; Marenich, A. V.; Bloino, J.; Janesko, B. G.; Gomperts, R.; Mennucci, B.; Hratchian, H. P.; Ortiz, J. V.; Izmaylov, A. F.; Sonnenberg, J. L.; Williams-Young, D.; Ding, F.; Lipparini, F.; Egidi, F.; Goings, J.; Peng, B.; Petrone, A.; Henderson, T.; Ranasinghe, D.; Zakrzewski, V. G.; Gao, J.; Rega, N.; Zheng, G.; Liang, W.; Hada, M.; Ehara, M.; Toyota, K.; Fukuda, R.; Hasegawa, J.; Ishida, M.; Nakajima, T.; Honda, Y.; Kitao, O.; Nakai, H.; Vreven, T.; Throssell, K.; Montgomery, J. A., Jr.; Peralta, J. E.; Ogliaro, F.; Bearpark, M. J.; Heyd, J. J.; Brothers, E. N.; Kudin, K. N.; Staroverov, V. N.; Keith, T. A.; Kobayashi, R.; Normand, J.; Raghavachari, K.; Rendell, A. P.; Burant, J. C.; Iyengar, S. S.; Tomasi, J.; Cossi, M.; Millam, J. M.; Klene, M.; Adamo, C.; Cammi, R.; Ochterski, J. W.; Martin, R. L.; Morokuma, K.; Farkas, O.; Foresman, J. B.; Fox, D. J. Gaussian, Inc., Wallingford CT, 2016.

47. GaussView, Version 6, Dennington, Roy; Keith, Todd A.; Millam, John M. Semichem Inc., Shawnee Mission, KS, 2016.

48. Stewart, J. J. P. Optimization of parameters for semiempirical methods VI: more modifications to the NDDO approximations and re-optimization of parameters. J. Mol. Model. 2013, 19, 1-32.

49. Yu, S.; Kumar, P.; Ward, J.S.; Frontera, A.; Rissanen, K. A "nucleophilic" iodine in a halogen-bonded iodonium complex mani-

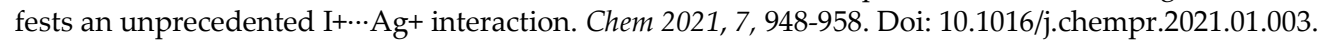

